\title{
Multiscale Asymptotics Analysis for the Mesoscale Dynamics of Cloud-Topped Boundary Layers
}

\author{
ANTONY Z. OWINOH \\ FB Mathematik und Informatik, Freie Universität Berlin, Berlin, Germany \\ BJORN STEVENS \\ Max-Planck-Institut für Meteorologie, Hamburg, Germany, and Department of Atmospheric \\ and Oceanic Sciences, University of California, Los Angeles, Los Angeles, California \\ RUPERT KLEIN \\ FB Mathematik und Informatik, Freie Universität Berlin, Berlin, Germany
}

(Manuscript received 10 February 2010, in final form 18 September 2010)

\begin{abstract}
This paper presents the derivation of a model to explore the coupling between the dynamic and thermodynamic processes of a cloud-topped boundary layer on mesoscales using a formal multiscale asymptotic approach. The derived equations show how the anomalies in the heat, moisture, and mass budgets in the boundary layer affect boundary layer motions, and how these motions can organize and amplify (or damp) such anomalies.

The thermodynamics equations are similar to those that have been suggested in mixed layer studies; that is, the evolution of the thermodynamics variables depends on the surface heat and moisture fluxes, cloud-top radiative cooling rate, temperature, and moisture jumps across the capping inversion. However, these equations are coupled to the dynamics equation through the entrainment rate at the top of the cloud deck. The entrainment rate is parameterized from results obtained in laboratory experiments and clearly shows the dependence on the velocity perturbation, which in turn strongly depends on the horizontal gradient of the thermodynamics variables. The derived entrainment rate is applicable when the thermal jump at cloud top is sufficiently weak and the velocity jump is on the order of the velocity perturbation.

Aside from some initial analyses of the main balances in steady-state solutions, the mathematical properties and physical characteristics of the system of equations will be explored in future papers.
\end{abstract}

\section{Introduction}

The atmospheric boundary layer energetically couples the atmosphere to the underlying surface, both directly through its regulation of the transfer of heat, momentum, and matter (e.g., water vapor) and indirectly through the modulation of radiative fluxes. Boundary layer processes thus readily imprint themselves on larger-scale circulations. For instance, boundary layer processes translate surface temperature gradients into shallow pressure anomalies that drive regions of low-level convergence

Corresponding author address: Dr. Antony Z. Owinoh, FB Mathematik und Informatik, Freie Universität Berlin, Arnimallee 6, 14195 Berlin, Germany.

E-mail: owinoh@mi.fu-berlin.de and, hence, the climatology of precipitation (Lindzen and Nigam 1987). Boundary layer processes also determine the distribution of low-level clouds that play such a crucial role in limiting the amount of radiant energy reaching the surface ocean. For these, and similar reasons, the study of boundary layer processes, and the development of theories or models capable of encapsulating them, is a topic of enduring interest.

Bulk, or integral, models play a special role in the study of boundary layer processes. Bulk models do not resolve the vertical structure of the boundary layer but rather predict the evolution of integral quantities such as the boundary layer budgets of boundary layer mass, momentum, energy, and perhaps material quantities as well. Such models are useful in their own right. They also provide a framework for understanding the behavior 
of more complex models. Of the variety of bulk models that have been proposed (Stevens et al. 2005), a particularly interesting one is the mixed layer model of Lilly (1968), as this provides an elegant framework for coupling the diversity of physical processes thought to control the distribution of marine stratiform cloudiness within the marine boundary layer.

Like many bulk models, the mixed layer model of Lilly is usually justified by assuming that the processes within the boundary layers are occurring on spatial scales much smaller, and temporal scales much shorter, than the scales of processes within the environment in which they are embedded. For instance, the large-scale divergence, which plays an important role in controlling boundary layer depth, or sea surface temperature gradients, which may generate boundary layer pressure gradients, are assumed to be decoupled from processes within the boundary layer. As a result, most studies with Lilly's mixed layer theory take on an essentially thermodynamic character as they focus on the budgets of thermal energy, moisture, and mass and their controls on cloud amount without exploring how the development of clouds, or cloud-scale processes, couples with mesoscale fluid motions within the boundary layer. To the extent that bulk models have been coupled to the dynamical evolution of the layer, the emphasis has been on the interaction between boundary layer processes and much larger-scale circulations (Schubert et al. 1979).

The interplay between dynamics and thermodynamic anomalies on a more intermediate scale is an issue that interests us. Specifically, we wish to develop a simplified theoretical framework applicable to such issues. Such a framework could help answer questions such as whether the local development of precipitation within the cloud layer perturbs the flow in a manner that reinforces the conditions that lead to the precipitation in the first place, or whether the local development of cloudiness generates flow anomalies that support the development of further cloudiness.

We adopt a formal asymptotic approach that admits a multiscale analysis. Our approach is based on a unified mathematical framework for the derivation of reduced multiscale models of geophysical flows, suggested by Klein (2004). The framework involves four key steps. First, the $3 \mathrm{D}$ compressible flow equations on the rotating sphere are made nondimensional through the identification of characteristic scales. Second, universal nondimensional parameters are identified that are independent of any specific flow phenomenon considered. Third, distinguished limits between these parameters are chosen. A summary of the distinguished limits and other assumptions are listed in Table 1. Finally, multiple-scale asymptotic expansions based on the small perturbation parameter are carried out. An initial attempt to evaluate the approach is made by exploring its ability to reproduce various wellknown (single scale) equations in geophysical fluid dynamics. Such an evaluation strategy has proven to be an important first step in the evaluation of the derivation of a variety of new multiscale models in the past (for example, in Majda and Klein 2003; Klein 2004; Biello and Majda 2005; Mikusky 2007; Dolaptchiev and Klein 2009), and including the case of boundary layer flows in the absence of cloud processes (Klein et al. 2005).

This paper presents the derivation of a model that admits coupling between dynamic and thermodynamic processes on intermediate scales. The derivation is based on two types of principal assumptions: asymptotic scaling assumptions and additional ones relating to parameterization closures. These assumptions are summarized in Table 1. Although focused on a particular regime, our asymptotic analysis addresses many of the more general issues associated with the coupling of moisture, phase changes, and the complications they entail to fluiddynamical processes. For the regime that we identify our asymptotic analysis shows that

- the thermodynamics variables are coupled to the dynamics through the pressure and entrainment terms;

- the velocity perturbations enter the thermodynamics equations through the entrainment rate and surface fluxes;

- the coupling between the two thermodynamics equations is due mainly to diabatic processes such as entrainment, radiative, and precipitation effects.

The analysis also shows that shallow-water-like wave dynamics appear if the thermal stratification capping the boundary layer is weak. This might mean that such wavelike dynamics is not important for most stratocumulus boundary layers, or that it only becomes important on larger space and longer time scales, which might be resolvable in the larger-scale models that are often forced to parameterize boundary layer processes as being essentially homogeneous over scales of hundreds of kilometers.

The outline of our presentation is as follows. Section 2 describes basic equations in dimensionless form with the appropriate space and time scales given in section 3 . The bulk evolution equations for momentum, energy, and moisture are then derived in section 4 . The equations include the surface and entrainment fluxes and other sources such as radiative and precipitation effects, which are then derived asymptotically in section 5. A summary of systems of equations based on the parameterizations is presented and discussed in section 6 for a weak 
TABLE 1. The model assumptions: symbols are defined in Tables 2-4.

i. Distinguished limits based on dimensionless Mach $M$, Froude Fr, and Rossby $\operatorname{Ro}_{h_{\mathrm{sc}}}$ numbers:

$M=\frac{u_{\mathrm{ref}}}{\sqrt{p_{\mathrm{ref}} / \rho_{\mathrm{ref}}}} \sim \varepsilon^{2}, \mathrm{Fr}=\frac{u_{\mathrm{ref}}}{\sqrt{g h_{\mathrm{sc}}}} \sim \varepsilon^{2}, \mathrm{Ro}_{h_{\mathrm{sc}}}=\frac{u_{\mathrm{ref}}}{\Omega h_{\mathrm{sc}}} \sim \varepsilon^{-1}$.

ii. Distinguished limits that emerge from moist thermodynamics:

$\frac{L_{v} \rho_{\text {ref }}}{p_{\text {ref }}} \equiv \varepsilon^{-1} L_{v}^{*}, \frac{R_{v}}{R_{d}} \equiv R^{*} \varepsilon^{0}, \frac{R_{d}}{c_{p d}} \equiv \Gamma^{*} \varepsilon, \frac{c_{l}}{c_{p d}} \equiv c_{p}^{*} \varepsilon^{-1}, \frac{p_{s, \text { ref }}}{p_{\text {ref }}} \equiv \varepsilon^{3 / 2} p_{s}^{*}, \frac{L_{v}}{R_{v} \theta_{\text {ref }}} \equiv \varepsilon^{-1} A^{*}$.

In obtaining these distinguished limits the temperature or pressure dependencies in thermodynamic parameters have been neglected. iii. The asymptotic expansions for the flow variables are given in (26a)-(26e), based on field observations.

iv. Closure assumptions

(a) No sources of heat are accounted for, other than the longwave radiative fluxes.

(b) Precipitation is the only source of moisture considered in the analysis.

(c) Saturation vapor pressure can be approximated by Clausius-Clapeyron equation.

(d) The bulk transfer formulas are used to parameterize the surface fluxes.

(e) Entrainment rate formula is based interfacial velocity jump and the bulk Richardson number.

Most of these closure assumptions are made to simplify the mathematical model and the neglected effects (e.g., shortwave radiation, evaporation of precipitation) can always be reintroduced parametrically.

v. Distinguished limits that emerge from closure assumptions:

$C_{D, H, Q} \equiv \varepsilon^{3} C^{*}, C_{E} \equiv \varepsilon C_{E}^{*}, a \equiv \varepsilon^{-5 / 2} a^{*}$.

buoyancy jump and weak surface fluxes. Our emphasis throughout is on the derivation of the model equations, but we also present some steady-state results in section 6. Detailed mathematical properties and physical characteristics of the ensuing equations will be explored in future work.

\section{Governing equations}

Our starting point is the fully compressible gas dynamics equations, for which Klein (2004) introduced the distinguished limit such that

$$
\begin{aligned}
\varepsilon \rightarrow 0: M & =\frac{u_{\text {ref }}}{\sqrt{p_{\text {ref }} / \rho_{\text {ref }}}} \sim \varepsilon^{2} ; \quad \text { Fr }=\frac{u_{\text {ref }}}{\sqrt{g h_{\mathrm{sc}}}} \sim \varepsilon^{2} ; \\
\operatorname{Ro}_{h_{\mathrm{sc}}} & =\frac{u_{\mathrm{ref}}}{\Omega h_{\mathrm{sc}}} \sim \varepsilon^{-1},
\end{aligned}
$$

where the Mach $M$, Froude Fr, and Rossby $\operatorname{Ro}_{h_{\mathrm{sc}}}$ numbers are the dimensional numbers defined in terms of the reference pressure $p_{\text {ref }}=10^{5} \mathrm{~m}^{-1} \mathrm{~s}^{-2}$, reference density $\rho_{\text {ref }}=1.25 \mathrm{~kg} \mathrm{~m}^{-3}$, pressure scale height $h_{\mathrm{sc}}=$ $p_{\text {ref }} / g \rho_{\text {ref }} \approx 10 \mathrm{~km}$, characteristic speed $u_{\text {ref }}=10 \mathrm{~m} \mathrm{~s}^{-1}$, reference temperature $\theta_{\text {ref }}=300 \mathrm{~K}$, characteristic time $t_{\text {ref }}=h_{\mathrm{sc}} / u_{\mathrm{ref}}=10^{3} \mathrm{~s}$, earth's rotation frequency $\Omega \sim$ $10^{-4} \mathrm{~s}^{-1}$, and gravitational acceleration $g=9.8 \mathrm{~m}^{-1} \mathrm{~s}^{-2}$. Frequently used symbols are listed in Tables 2-4 for subsequent reference.

Please note that, the appropriate Rossby number $\operatorname{Ro}_{L}$ for most atmospheric flow phenomena involves the horizontal rather than the vertical length scale as used here. This is obtained from $\mathrm{Ro}_{h_{\mathrm{sc}}}$ by an appropriate rescaling with the relevant aspect ratio. For example, if one was interested in a flow on a horizontal length scale $L=\varepsilon^{\alpha} h_{\mathrm{sc}}$, then the Rossby number for the motion would simply be $\mathrm{Ro}_{L}=\varepsilon^{-\alpha} \mathrm{Ro}_{h_{\mathrm{sc}}}$. If $L$ was a synoptic length scale with $L=\varepsilon^{-2} h_{\mathrm{sc}}$, then one obtains $\operatorname{Ro}_{L}=O(\varepsilon) \ll 1$ as expected.

One may think of $\varepsilon$ as a parameter measuring the ratio of the gravitational versus angular accelerations:

$$
\varepsilon=\sqrt[3]{\frac{a \Omega}{g}} \sim \frac{1}{8} \cdots \frac{1}{6}
$$

with the earth's radius $a \sim 6000 \mathrm{~km}$. In physical problems with more than one small parameter, it is known that the asymptotic equations depend strongly on the path by which the parameters approach their respective limiting values. Thus, to remove this dependence one requires a limit by which the parameters are related to one another. Of course, different distinguishing limits are possible, but the emergence of a wide family of meteorological equations from one and the same distinguished limit as a starting point provides a posteriori support for the distinguished limit given by (1); see Klein (2010) and references therein. Table 5 identifies the order of magnitudes that arise from an $\varepsilon$ expansion of different variables that appear in this analysis.

In the following, we adopt a Cartesian coordinate system $\mathbf{x}=(x, y, z)$ rotating with angular velocity $\boldsymbol{\Omega}$, with gravity $g$ acting in the (vertical) $z$ direction. The rotation vector $\boldsymbol{\Omega}$ is assumed to take a constant value, consistent with a tangent plane approximation. If $\rho(\mathrm{x}, t)$ and $\mathbf{v}(\mathbf{x}, t)=\mathbf{v}_{\|}+w \mathbf{k}$ denote the fluid density and velocity 
TABLE 2. Summary of symbols.

\begin{tabular}{|c|c|}
\hline Symbol & Meaning \\
\hline$c_{p d}$ & $\begin{array}{l}\text { Specific heat capacity at constant pressure } \\
\text { of dry air }\left(1007 \mathrm{~J} \mathrm{~kg}^{-1} \mathrm{~K}^{-1}\right)\end{array}$ \\
\hline$c_{l}$ & Heat capacity of liquid water $\left(4217 \mathrm{~J} \mathrm{~kg}^{-1} \mathrm{~K}^{-1}\right)$ \\
\hline$g$ & Gravity $\left(9.8 \mathrm{~m} \mathrm{~s}^{-1}\right)$ \\
\hline$h_{\mathrm{sc}}$ & Pressure scale height $(10 \mathrm{~km})$ \\
\hline$p$ & Pressure \\
\hline$q_{v}$ & Water vapor mixing ratio \\
\hline$q_{l}$ & Liquid water mixing ratio \\
\hline$q_{s}$ & Saturation mixing ratio \\
\hline$q_{t}$ & Total water mixing ratio \\
\hline$t$ & Time \\
\hline $\mathbf{v}_{\|}$ & Horizontal velocities $(u, v)$ \\
\hline$w$ & Vertical velocity \\
\hline$z$ & Height above the surface \\
\hline$C_{D}$ & Bulk transfer coefficient of momentum \\
\hline$C_{E}$ & Entrainment rate coefficient \\
\hline$C_{H}$ & Bulk transfer coefficient of heat \\
\hline$C_{Q}$ & Bulk transfer coefficient of moisture \\
\hline$C_{R}$ & Precipitation conversion rate \\
\hline$E$ & Cloud-top entrainment velocity \\
\hline$H$ & Depth of the boundary layer \\
\hline$L_{v}$ & Latent heat of vaporization $\left(2.47 \mathrm{MJ} \mathrm{kg}^{-1}\right)$ \\
\hline$R_{d}$ & Gas constant for dry air $\left(287.0 \mathrm{~J} \mathrm{~kg}^{-1} \mathrm{~K}^{-1}\right)$ \\
\hline$R_{v}$ & Gas constant for water vapor $\left(461.5 \mathrm{~J} \mathrm{~kg}^{-1} \mathrm{~K}^{-1}\right)$ \\
\hline$F_{L}^{\uparrow \downarrow}(z)$ & $\begin{array}{l}\text { Upward and downward longwave radiative } \\
\text { flux at height } z\end{array}$ \\
\hline$T$ & $\begin{array}{l}\text { Time associated with the horizontal } \\
\text { advection }(\sim 2 \mathrm{~h})\end{array}$ \\
\hline $\mathrm{S}_{\theta_{e}}$ & $\begin{array}{l}\text { Source term in the equivalent potential } \\
\text { temperature equation }\end{array}$ \\
\hline $\mathcal{S}_{q}$ & Source term in the total water mixing ratio equation \\
\hline$\beta^{q_{t}}$ & $\begin{array}{l}\text { Free tropospheric gradient of equivalent } \\
\text { potential temperature }\end{array}$ \\
\hline$\beta_{1}$ & $\begin{array}{l}\text { Gradient of liquid water mixing ratio } \\
\text { in the cloud layer }\end{array}$ \\
\hline$\delta$ & Asymptotic scaling parameter $(\delta=\sqrt{\varepsilon})$ \\
\hline$\epsilon$ & Emissivity \\
\hline$\varepsilon$ & Asymptotic scaling parameter \\
\hline$\eta$ & Height scaled with the depth of the boundary layer \\
\hline$\eta_{c}$ & Cloud-base height \\
\hline$\theta_{e}$ & Equivalent potential temperature \\
\hline$\rho$ & Density \\
\hline$\varphi^{(i)}$ & Given by (B8) \\
\hline$\tau$ & $\begin{array}{l}\text { Time associated with convective time } \\
\text { scale }(\sim 20 \mathrm{~min})\end{array}$ \\
\hline$\xi$ & Length scaled with $600 \mathrm{~m}$ \\
\hline$\widehat{\Omega}$ & Normalized earth rotation vector \\
\hline
\end{tabular}

fields at position $\mathbf{x}=\mathbf{x}_{\|}+z \mathbf{k}$ and time $t$, the mass conservation equation is

$$
\frac{\partial \rho}{\partial t}+\nabla_{\|} \cdot\left(\rho \mathbf{v}_{\|}\right)+\frac{\partial}{\partial z}(\rho w)=0
$$

In conservation form, the horizontal component of the momentum equation is
TABLE 3. Summary of subscripts, superscripts, and operators relative to a variable $\phi$.

\begin{tabular}{ll}
\hline \hline Symbol & \multicolumn{1}{c}{ Meaning } \\
\hline$\phi_{\text {ref }}$ & Reference quantity of $\phi$ \\
$\phi_{0}$ & Surface values \\
$\phi^{(i)}$ & The $\delta^{i}$ th coefficient in the asymptotic \\
& expansion for $\phi$
\end{tabular}

$$
\begin{aligned}
& \frac{\partial}{\partial t}\left(\rho \mathbf{v}_{\|}\right)+\nabla_{\|} \cdot\left(\rho \mathbf{v}_{\|} \cdot \mathbf{v}_{\|}\right)+\frac{\partial}{\partial z}\left(\rho \mathbf{v}_{\|} w\right)+\varepsilon(\widehat{\mathbf{\Omega}} \times \rho \mathbf{v})_{\|} \\
& \quad+\frac{1}{\varepsilon^{4}} \boldsymbol{\nabla}_{\|} p=0
\end{aligned}
$$

and the vertical component is given by

$$
\begin{aligned}
& \frac{\partial}{\partial t}(\rho w)+\nabla_{\|} \cdot\left(\rho \mathbf{v}_{\|} w\right)+\frac{\partial}{\partial z}(\rho w w)+\varepsilon(\widehat{\mathbf{\Omega}} \times \rho \mathbf{v})_{\perp} \\
& \quad+\frac{1}{\varepsilon^{4}} \frac{\partial p}{\partial z}=-\frac{1}{\varepsilon^{4}} \rho .
\end{aligned}
$$

The anisotropy between horizontal and vertical motions, associated with the volumetric force due to gravity, which appears on the rhs of (5), motivates our separate treatment of the horizontal versus vertical component of momentum.

The heat and moisture budgets are described by conservation laws for the equivalent potential temperature (Emanuel 1994) and total water mixing ratio:

TABLE 4. Summary of order one parameters.

\begin{tabular}{cll}
\hline Symbol & \multicolumn{1}{c}{ Meaning } & \multicolumn{1}{c}{$\begin{array}{c}\text { Introductory } \\
\text { equation }\end{array}$} \\
\hline$a^{*}$ & $\begin{array}{c}\text { Extinction cross section of the } \\
\text { liquid water } \\
\end{array}$ & Below (67) \\
$c_{p}^{*}$ & Specific heat capacity ratio \\
$p_{s}^{*}$ & Saturation vapor pressure \\
$A^{*}$ & Latent heat of vaporization, & $(10)$ \\
& $\quad$ scaled with $R_{v} \theta_{\text {ref }}$ & Below (A27) \\
$C^{*}$ & Bulk transfer coefficient & Below (79) \\
$C_{E}^{*}$ & Entrainment rate coefficient & Below (92) \\
$L_{v}^{*}$ & Latent heat of vaporization & $(10)$ \\
& $\quad$ scaled with $R_{d} \theta_{\text {ref }}$ \\
$R^{*}$ & Ratio of gas constants $R_{v} / R_{d}$ & $(10)$ \\
$\mathcal{P}^{*}$ & Precipitation conversion rate & $(77)$ \\
$\Gamma^{*}$ & Isentropic exponent & $(10)$ \\
\hline
\end{tabular}


TABLE 5. Dimensional magnitudes (with varying but convenient units) for reference quantities for $\varepsilon=1 / 7$.

\begin{tabular}{cccccl}
\hline \hline$\alpha$ & $\varepsilon^{\alpha}$ & $\varepsilon^{\alpha} u_{\text {ref }}$ & $\varepsilon^{\alpha} h_{\text {ref }}$ & $\varepsilon^{\alpha} \theta_{\text {ref }}$ & $\varepsilon^{\alpha} t_{\text {ref }}$ \\
\hline-3 & 345 & & $3450 \mathrm{~km}$ & & $4 \mathrm{~d}$ \\
$-5 / 2$ & 130 & & $1296 \mathrm{~km}$ & & $1.5 \mathrm{~d}$ \\
-2 & 49 & & $490 \mathrm{~km}$ & & $14 \mathrm{~h}$ \\
$-3 / 2$ & 19 & $185 \mathrm{~m} \mathrm{~s}^{-1}$ & $185 \mathrm{~km}$ & & $5 \mathrm{~h}$ \\
-1 & 7 & $70 \mathrm{~m} \mathrm{~s}^{-1}$ & $70 \mathrm{~km}$ & & $2 \mathrm{~h}$ \\
$-1 / 2$ & 2.65 & $26 \mathrm{~m} \mathrm{~s}^{-1}$ & $26 \mathrm{~km}$ & & $44 \mathrm{~min}$ \\
0 & 1 & $10 \mathrm{~m} \mathrm{~s}^{-1}$ & $10 \mathrm{~km}$ & $300 \mathrm{~K}$ & $17 \mathrm{~min}$ \\
$1 / 2$ & 0.38 & $3.78 \mathrm{~m} \mathrm{~s}^{-1}$ & $3780 \mathrm{~m}$ & $113 \mathrm{~K}$ & $6 \mathrm{~min}$ \\
1 & 0.14 & $1.43 \mathrm{~m} \mathrm{~s}^{-1}$ & $1429 \mathrm{~m}$ & $43 \mathrm{~K}$ & $2 \mathrm{~min}$ \\
$3 / 2$ & 0.054 & $0.54 \mathrm{~m} \mathrm{~s}^{-1}$ & $540 \mathrm{~m}$ & $16 \mathrm{~K}$ & $54 \mathrm{~s}$ \\
2 & 0.020 & $0.20 \mathrm{~m} \mathrm{~s}^{-1}$ & $204 \mathrm{~m}$ & $6.1 \mathrm{~K}$ & $20 \mathrm{~s}$ \\
$5 / 2$ & 0.0077 & $7.71 \mathrm{~cm} \mathrm{~s}^{-1}$ & $77 \mathrm{~m}$ & $2.3 \mathrm{~K}$ & $8 \mathrm{~s}$ \\
3 & 0.0029 & $2.92 \mathrm{~cm} \mathrm{~s}^{-1}$ & $29 \mathrm{~m}$ & $0.87 \mathrm{~K}$ & $3 \mathrm{~s}$ \\
$7 / 2$ & 0.0011 & $1.10 \mathrm{~cm} \mathrm{~s}^{-1}$ & $11 \mathrm{~m}$ & $0.33 \mathrm{~K}$ & $1 \mathrm{~s}$ \\
4 & 0.0004 & $0.42 \mathrm{~cm} \mathrm{~s}^{-1}$ & $4 \mathrm{~m}$ & $0.12 \mathrm{~K}$ & $0.4 \mathrm{~s}$ \\
\hline
\end{tabular}

$$
\frac{\partial}{\partial t}\left(\rho \theta_{e}\right)+\nabla_{\|} \cdot\left(\rho \mathbf{v}_{\|} \theta_{e}\right)+\frac{\partial}{\partial z}\left(\rho w \theta_{e}\right)=\rho \mathcal{S}_{\theta_{e}}
$$

and

$$
\frac{\partial}{\partial t}\left(\rho q_{t}\right)+\nabla_{\|} \cdot\left(\rho \mathbf{v}_{\|} q_{t}\right)+\frac{\partial}{\partial z}\left(\rho w q_{t}\right)=\rho \mathcal{S}_{q_{t}},
$$

respectively. The source term $\mathcal{S}_{\theta_{e}}$ represents diabatic processes, for instance, radiation, as described in section 5b. The term $\mathcal{S}_{q_{t}}$ represents the net moisture addition (or removal) rate, for instance, as a result of precipitation.

Equations (3)-(7) are closed given an equation of state, which we take to be that of an ideal mixture of water vapor and dry air. Here we write it in terms of the equivalent potential temperature and our small parameter $\varepsilon$ :

$$
\begin{aligned}
\rho \theta_{e}= & \left(1+q_{t}\right)\left[\frac{p}{\left(1+R^{*} q_{v}\right)}\right]^{\left[1-\varepsilon \Gamma_{\varepsilon}\right]}\left(\frac{q_{s}}{q_{v}}\right)^{\varepsilon \Gamma_{\varepsilon} R^{*} q_{v}} \\
& \times \exp \left[L_{v}^{*} \frac{\left(1+R^{*} q_{v}\right)}{\left(1+q_{t}\right)} \frac{\rho q_{v}}{p}\right] .
\end{aligned}
$$

In deriving (8) we have introduced the abbreviation

$$
\Gamma_{\varepsilon}=\frac{\Gamma^{*}}{1+\varepsilon^{-1} c_{p}^{*} q_{t}}
$$

and have extended the distinguished limits in (1) to incorporate dimensionless thermodynamic numbers so that

$$
\begin{aligned}
\frac{L_{v} \rho_{\mathrm{ref}}}{p_{\mathrm{ref}}} & \equiv \varepsilon^{-1} L_{v}^{*} ; \quad \frac{R_{v}}{R_{d}} \equiv R^{*} \varepsilon^{0} ; \quad \frac{R_{d}}{c_{p d}} \equiv \Gamma^{*} \varepsilon ; \\
\frac{c_{l}}{c_{p d}} & \equiv c_{p}^{*} \varepsilon^{-1},
\end{aligned}
$$

where $\Gamma^{*}, L_{v}^{*}, R^{*}$, and $c_{p}^{*}$ are on the order of unity. These terms are obtained by choosing typical values of $L_{v}, R_{d}, R_{v}, c_{l}$, and $c_{p d}$ (see Table 2) and writing the expressions in (10) as orders of magnitude in terms of $\varepsilon$. Temperature or pressure dependencies in the original thermodynamic parameters have been neglected. This is justified since, for example, $L_{v}=2.50 \mathrm{MJ} \mathrm{kg}^{-1}, c_{l}=$ $1005.16 \mathrm{~J} \mathrm{~kg}^{-1} \mathrm{~K}^{-1}$, and $c_{p d}=4178 \mathrm{~J} \mathrm{~kg}^{-1} \mathrm{~K}^{-1}$ at $0^{\circ} \mathrm{C}$ and increasing the temperature to, say, $40^{\circ} \mathrm{C}$ yields $L_{v}=$ $2.41 \mathrm{MJ} \mathrm{kg}^{-1}, c_{l}=1006.19 \mathrm{~J} \mathrm{~kg}^{-1} \mathrm{~K}^{-1}$, and $c_{p d}=$ $4178 \mathrm{~J} \mathrm{~kg}^{-1} \mathrm{~K}^{-1}$. The full derivation and justification of (8) is provided in the appendix A (see also Klein and Majda 2006). For now it is sufficient to note that, while the complexity of (8) results from our retention of all the terms in the definition of $\theta_{e}$, it adds nothing of substance to the leading-order systems of equations that we derive: one contribution of this work is to demonstrate this point, which can be readily extended to analogous systems of equations in more common usage.

As we explore the implications of the distinguished limit introduced through (1) and (10) we shall find that we arrive at equations that are incomplete, or not closed. Closure requires models for the representation of smallscale fluid dynamical processes such as surface and entrainment fluxes, and non-fluid-dynamical processes such as radiative transfer or cloud microphysical interactions. Even the simplest models that one proposes to represent such processes, and hence close our equations, introduce additional parameters that must be scaled and cast in the framework of our distinguished limit. So doing complicates the analysis and further illustrates the challenge of developing an asymptotically consistent ansatz for atmospheric flows involving cloud processes.

In summary, (3)-(8) define a closed system of equations under the distinguished limit given by (1) and (10). They form the starting point for our subsequent analysis. The equations themselves are standard; however, the limit, and its elaboration through the incorporation of additional physical processes, is not. Our hypothesis is that the distinguished limit that we introduce captures essential asymptotic behavior of the real system, and thus is meaningful.

\section{Spatial and temporal scales}

Stratocumulus evince structure on a wide range of spatial and temporal scales, particularly under the influence of remotely generated gravity waves or in the presence of diabatic processes such as precipitation (Savic-Jovcic and Stevens 2007). Here we explore the coupling of thermodynamic and dynamic processes on the mesoscale, which we define to be a horizontal scale of about $70 \mathrm{~km}$ (i.e., $\varepsilon^{-1} h_{\mathrm{sc}}$ ). These scales are much 
smaller than those typically resolved by large-scale models but much larger than the scales typically associated with the energetic eddies of the boundary layer itself. The latter scale with the boundary layer height, which we take to be $500-600 \mathrm{~m}$ (i.e., $\varepsilon^{3 / 2} h_{\mathrm{sc}}$ ). Although the cloud base height could potentially appear as another independent length scale, we will rather extract it from the thermodynamics later in section $5 \mathrm{a}$ as a consequence of the present scalings.

We consider time scales associated with the horizontal advection $\varepsilon^{-1} t_{\text {ref }}(\sim 2 \mathrm{~h})$ and convective time scale $t_{\text {ref }}$ ( $\sim 20 \mathrm{~min})$, assuming a convective velocity $O\left(0.5 \mathrm{~m} \mathrm{~s}^{-1}\right)$ $\left(\varepsilon^{3 / 2} u_{\text {ref }}\right)$ and based on the short length scale $\varepsilon^{3 / 2} h_{\mathrm{sc}}$. Thus, we will seek asymptotic solutions in terms of the new multiple-scale coordinate system: $\mathbf{X}=\varepsilon \mathbf{x}_{\|}, \xi=$ $\varepsilon^{-3 / 2} \mathbf{x}_{\|}, \eta=\varepsilon^{-3 / 2} z, T=\varepsilon t$, and $\tau=t$. In these expressions $\eta$ is the scaled vertical coordinate, whereas $\xi, \tau$ are the fast and $X, T$ the slow variables for the horizontal directions and time, respectively. Based on these scales and in terms of $\delta=\varepsilon^{1 / 2}$, the governing equations (3)-(7) rescale to

$$
\delta^{3}\left(\frac{\partial}{\partial \tau}+\delta^{2} \frac{\partial}{\partial T}\right) \rho+\left(\nabla_{\xi}+\delta^{5} \nabla_{X}\right) \cdot\left(\rho \mathbf{v}_{\|}\right)+\frac{\partial}{\partial \eta}(\rho w)=0,
$$

$$
\begin{aligned}
& \delta^{3}\left(\frac{\partial}{\partial \tau}+\delta^{2} \frac{\partial}{\partial T}\right)\left(\rho \mathbf{v}_{\|}\right)+\left(\nabla_{\xi}+\delta^{5} \nabla_{X}\right) \cdot\left(\rho \mathbf{v}_{\|} \cdot \mathbf{v}_{\|}\right) \\
& +\frac{\partial}{\partial \eta}\left(\rho \mathbf{v}_{\|} w\right)+\delta^{5}(\widehat{\boldsymbol{\Omega}} \times \rho \mathbf{v})_{\|}+\frac{1}{\delta^{8}}\left(\nabla_{\xi} p+\delta^{5} \nabla_{X} p\right)=0,
\end{aligned}
$$

$$
\begin{aligned}
& \delta^{3}\left(\frac{\partial}{\partial \tau}+\delta^{2} \frac{\partial}{\partial T}\right)(\rho w)+\left(\nabla_{\xi}+\delta^{5} \nabla_{X}\right) \cdot\left(\rho \mathbf{v}_{\|} w\right) \\
& +\frac{\partial}{\partial \eta}(\rho w w)+\delta^{5}(\widehat{\boldsymbol{\Omega}} \times \rho \mathbf{v})+\frac{1}{\delta^{8}}\left(p_{\eta}+\delta^{3} \rho\right)=0,
\end{aligned}
$$

$$
\begin{aligned}
& \delta^{3}\left(\frac{\partial}{\partial \tau}+\delta^{2} \frac{\partial}{\partial T}\right)\left(\rho \theta_{e}\right)+\left(\nabla_{\xi}+\delta^{5} \nabla_{X}\right) \cdot\left(\rho \mathbf{v}_{\|} \theta_{e}\right) \\
& +\frac{\partial}{\partial \eta}\left(\rho w \theta_{e}\right)=\delta^{3} \rho \mathcal{S}_{\theta_{e}},
\end{aligned}
$$

and

$$
\begin{aligned}
& \delta^{3}\left(\frac{\partial}{\partial \tau}+\delta^{2} \frac{\partial}{\partial T}\right)\left(\rho q_{t}\right)+\left(\nabla_{\xi}+\delta^{5} \nabla_{X}\right) \cdot\left(\rho \mathbf{v}_{\|} q_{t}\right) \\
& +\frac{\partial}{\partial \eta}\left(\rho w q_{t}\right)=\delta^{3} \rho \mathcal{S}_{q_{t}} .
\end{aligned}
$$

The parameter $\delta$ has been introduced instead of $\varepsilon$ so as to allow a more finely grained selection of scales.

\section{Averaging}

In this section we derive a new set of bulk, or vertically averaged, equations describing the leading-order balance of the intermediate scales selected for our analysis, with the fine and fast scales averaged over and parameterized. Equations (11)-(15), together with the equation of state (8) expressed in terms of $\delta$, are taken as a starting point. Three main steps are involved. First, we vertically average our equations; second, the dependent variables are expanded in terms of the small parameter $\delta$ and balances at different orders are identified; and, third, we average over the short spatial and fast temporal scales to derive the sublinear growth conditions that determine the large-scale, long-time evolution. Nonlinear terms that do not vanish under the averaging over fast scales are then identified and parameterizations of these terms are introduced in the subsequent section.

Vertical averaging of the equations introduces the concept of the boundary layer depth and processes that control it. We identify the boundary layer top as a semipermeable surface, whose height we denote by $H$. Vertical averaging also links the vertical momentum equation to the equation of state. Because the leadingorder balances are hydrostatic, vertical averaging of the vertical momentum provides a relationship between pressure and density within the boundary layer, given the pressure at $H, p_{H}$. Combining this with the equation of state provides a set of diagnostic relations for pressure and density at different orders, and the thermodynamic state of the boundary layer given by $\theta_{e}$ and $q_{t}$. Hence, as is familiar from bulk analyses (see, e.g., Schubert et al. 1979), one arrives at a new governing set of equations for the prognostic variables $\left\{H, \mathrm{v}_{\|}, \theta_{e}, q_{t}\right\}$, complemented by a set of diagnostic relations that describe (perturbations of) $p$ and $\rho$ as a function of $\theta_{e}, q_{t}, p_{H}$, and $\eta$.

In what follows we outline the basic steps involved and the technical difficulties insofar as they arise. Examples of how the analysis is performed are given for the mass balance equation and can be extended by the interested reader naturally to the case of the other equations. In so doing some technical difficulties arise in the treatment of the pressure gradient terms in the horizontal momentum equations. These issues, and how they are dealt with, are specifically addressed in a separate subsection.

\section{a. Depth averaging}

The equations are vertically integrated through the layer from the surface at $z=z_{0}(x, y)$ to a free surface $z=$ $H(x, y, t)+z_{0}(x, y)$. The lower boundary condition is $w=\mathbf{v}_{\|} \cdot \nabla z_{0}$ on $z=z_{0}(x, y)$ and the kinematic free 
surface condition in the absence of entrainment is $\partial H / \partial t=$ $\mathbf{v} \cdot \mathbf{n}$ on $\mathrm{z}=H(x, y, t)+z_{0}(x, y)$. The normal vector $\mathbf{n}=$ $-\nabla\left[z_{0}(x, y)+H(x, y, t)-z\right]$ points upward, and $\mathbf{v}_{\|}$and $\nabla_{\|}$denote the horizontal component of the velocity and gradient operator, respectively. Throughout we denote surface values by subscript 0 . In subsequent analysis we ignore the variation in the topography, that is, assume $z_{0}=0$.

The dimensionless free surface kinematic boundary conditions on $\eta=H$ is expressed as

$$
\delta^{3} \frac{\partial H}{\partial t}=(\mathbf{v}+\mathbf{E}) \cdot \mathbf{n},
$$

which introduces the entrainment velocity, $\mathbf{E}=E \mathbf{n}$, which encapsulates the permeability of the interface at $H$. Here $\mathbf{n}$ is the normal to the surface $\eta=H$ so that $\mathbf{n}=$ $\mathrm{k}-\delta^{3} \nabla_{\|} H$. In terms of the multiscale coordinates, (16) reads

$$
\begin{aligned}
& \delta^{3}\left(\frac{\partial}{\partial \tau}+\delta^{2} \frac{\partial}{\partial T}\right) H+\mathbf{v}_{\|} \cdot\left(\nabla_{\xi}+\delta^{5} \nabla_{\mathrm{x}}\right) H=(w+E) \text { on } \\
& \quad \eta=H .
\end{aligned}
$$

Since we assume a flat bottom, the lower boundary condition is given by

$$
w=0 \quad \text { on } \quad \eta=0
$$

We define the vertical average over the depth $H$ of some quantity $\phi$ as

$$
\langle\phi\rangle=\frac{1}{H} \int_{0}^{H} \phi d \eta .
$$

So, for example, averaging the continuity equation (11) and making use of the boundary condition in (18) results in a revised continuity equation, one that describes the overall mass balance in the layer of depth $H$ and makes explicit reference to the entrainment velocity $E$ :

$\delta^{3}\left(\frac{\partial}{\partial \tau}+\delta^{2} \frac{\partial}{\partial T}\right)(H\langle\rho\rangle)+\left(\nabla_{\xi}+\delta^{5} \nabla_{X}\right) \cdot\left(H\left\langle\rho \mathbf{v}_{\|}\right\rangle\right)=\rho_{H} E$.

\section{b. Leading-order equations}

The equations are now written in terms of dependent flow variables expanded in terms of the small parameter $\delta$. Thus, generically, for a dependent variable $\phi$ we write

$$
\phi=\sum_{i=0} \delta^{i} \phi^{(i)}(\tau, \xi, \eta, T, \mathbf{X}) .
$$

Applying this expansion to the mass continuity equation for the layer in (20) results in

$$
\begin{gathered}
\nabla_{\xi} \cdot\left(H\left\langle\rho \mathbf{v}_{\|}\right\rangle\right)^{(i)}=\left(\rho_{H} E\right)^{(i)} \quad \text { for } \quad i=0,1,2, \quad(22) \\
\frac{\partial}{\partial \tau}(H\langle\rho\rangle)^{(i-3)}+\nabla_{\xi} \cdot\left(H\left\langle\rho \mathbf{v}_{\|}\right\rangle\right)^{(i)}=\left(\rho_{H} E\right)^{(i)} \text { for } i=3,4,
\end{gathered}
$$

and

$$
\begin{aligned}
& \frac{\partial}{\partial \tau}(H\langle\rho\rangle)^{(i-3)}+\nabla_{\xi} \cdot\left(H\left\langle\rho \mathbf{v}_{\|}\right\rangle\right)^{(i)}=\left(\rho_{H} E\right)^{(i)} \\
& \quad-\left[\frac{\partial}{\partial T}(H\langle\rho\rangle)^{(i-5)}+\nabla_{X} \cdot\left(H\left\langle\rho \mathbf{v}_{\|}\right\rangle\right)^{(i-5)}\right] \\
& \text { for } i=5,6,7, \cdots .
\end{aligned}
$$

Here we note that the decomposition results in the initial equation being broken into a sequence of equations describing balances at different order. Compound terms of the form $(\phi \psi)^{(i)}$ are to be understood in terms of their component expansion such that

$$
(\phi \psi)^{(i)} \equiv \sum_{j=0}^{i} \phi^{(i-j)} \psi^{(j)},
$$

where here $\phi$ and $\psi$ denote two different dependent variables, for instance $H$ and $\mathbf{v}_{\|}$. Similar notation holds for terms involving more than two dependent variables.

Although (21) holds in general, for specific variables we will additionally assume that variability as a function of the independent variables only emerges at a specific order. So doing causes some terms to vanish at low order because, for instance, gradients in the balance equations are zero at that order. The assumptions we make are as follows:

$$
\begin{aligned}
\theta_{e}= & 1+\delta^{3} \theta_{e}^{(3)}+\delta^{4} \theta_{e}^{(4)}+\delta^{5} \theta_{e}^{(5)}+\delta^{6} \theta_{e}^{(6)}(\mathbf{X}, T) \\
& +\delta^{7} \theta_{e}^{(7)}(\mathbf{X}, \eta, T)+\delta^{8} \theta_{e}^{(8)}(\mathbf{X}, \xi, \eta, T, \tau)+\cdots \\
q_{t}= & \delta^{3} q_{t}^{(3)}+\delta^{4} q_{t}^{(4)}+\delta^{5} q_{t}^{(5)}+\delta^{6} q_{t}^{(6)}(\mathbf{X}, T) \\
& +\delta^{7} q_{t}^{(7)}(\mathbf{X}, \eta, T)+\delta^{8} q_{t}^{(8)}(\mathbf{X}, \xi, \eta, T, \tau)+\cdots
\end{aligned}
$$

and

$$
\begin{aligned}
q_{v}= & \delta^{3} q_{v}^{(3)}+\delta^{4} q_{v}^{(4)}+\delta^{5} q_{v}^{(5)}+\delta^{6} q_{v}^{(6)}(\mathbf{X}, \eta, T) \\
& +\delta^{7} q_{v}^{(7)}(\mathbf{X}, \eta, T)+\delta^{8} q_{v}^{(8)}(\mathbf{X}, \xi, \eta, T, \tau) \cdots
\end{aligned}
$$

We shall see later, especially from (B1)-(B3), that the absence of first- and second-order terms in the expression for $\theta_{e}$ follows directly from the equation of state and the hydrostatic balance that emerges at low order. The structure for $q_{v}$ and $q_{t}$ assumed (i.e., water vapor 
perturbations are small compared to unity). It will be shown in addition that the saturation vapor mixing ratio $q_{s}$ follows the form given for $q_{v}$.

For the boundary layer height we assume the following dependencies at various orders:

$$
\begin{aligned}
H= & H^{(0)}+\delta H^{(1)}(\mathbf{X}, T)+\delta^{2} H^{(2)}(\mathbf{X}, T) \\
& +\delta^{3} H^{(3)}(\mathbf{X}, \xi, T, \tau)+\cdots .
\end{aligned}
$$

Finally, we assume that

$$
\begin{aligned}
\mathbf{v}_{\|}= & \mathbf{v}_{\|}^{(0)}(\mathbf{X})+\delta \mathbf{v}_{\|}^{(1)}(\mathbf{X}, T)+\delta^{2} \mathbf{v}_{\|}^{(2)}(\mathbf{X}, T) \\
& +\delta^{3} \mathbf{v}_{\|}^{(3)}(\mathbf{X}, \xi, \eta, T, \tau)+\cdots .
\end{aligned}
$$

The assumptions on the scalings given in (26) are based on field observations and simulations (Stevens et al. 2003, 2005; Faloona et al. 2005). We will show later, for example from (48), that $\mathbf{v}_{\|}^{(0)}$ depends on the free atmosphere geostrophic pressure gradient and thus one can allow for variation in space $\mathbf{X}$ in $\mathbf{v}_{\|}^{(0)}$ so as to allow for large-scale vertical motion. By continuity this velocity scaling implies that the leading-order terms for $w$ vanish: that is, $w^{(i)}=0$ for $i<5$.

\section{c. Fast scale averaged equations}

We average the equations over fast temporal and small spatial scales; that is, we average over $\tau$ and $\xi$, respectively. Using the overbar to denote such averaging we have

$$
\bar{\psi}(\mathbf{X}, \eta, T)=\lim _{\tau, A \rightarrow \infty} \frac{1}{\tau A} \int_{\tau, A} \psi(\xi, \mathbf{X}, \eta, \tau, T) d \xi d \tau .
$$

Averaging over fast scales eliminates gradients on these scales due to the so-called sublinear growth condition so that, for instance, the leading-order terms of the mass balance equation become

$$
\bar{E}^{(i)}=0 \quad \text { for } \quad i=0,1,2,3,4,5
$$

and

$$
\begin{aligned}
& \frac{\partial H^{(1)}}{\partial T}+H^{(0)} \nabla_{X} \cdot \mathbf{v}_{\|}^{(1)}+H^{(1)} \nabla_{X} \cdot \mathbf{v}_{\|}^{(0)} \\
& +\mathbf{v}_{\|}^{(0)} \cdot \nabla_{X} H^{(1)}=\bar{E}^{(6)} .
\end{aligned}
$$

\section{d. Pressure gradients}

Averaging the horizontal momentum equation leads to terms of the form

$$
\int_{0}^{H} \nabla_{\xi} p d \eta \text { and } \delta^{5} \int_{0}^{H} \nabla_{X} p d \eta
$$

The finescale pressure gradients appear at lower order but are eliminated by the fast scale averaging. The largerscale pressure gradients must be evaluated. To do so we derive diagnostic equations for the pressure at the desired order starting with the equation of state and the vertically integrated vertical momentum equation, which remains hydrostatic on the scales of motion that interest us. The appropriate order of the pressure is then substituted into the above integrals and used to evaluate the vertically averaged pressure gradient terms. To make use of the vertically averaged momentum equation we will need a pressure boundary condition $P_{H}$.

\section{1) Pressure above the Boundary LAyer}

Consider a vertical scale greater than the boundary layer depth, that is, a scale on the order of the depth of the troposphere, $\sim 10 \mathrm{~km}$. We further assume that the nature of flow above the boundary layer is such that the horizontal scale remains $70 \mathrm{~km}$ or larger. Reduced equations with such scaling can be obtained using the asymptotic expansions in powers of $\varepsilon$ (or $\delta$ ). Asymptotic analysis of the continuity and momentum equations shows that the pressure in the layer is essentially hydrostatic. From the hydrostatic equation together with the equation of state, that is,

$$
p_{z}=-\rho \text { and } \rho \theta_{e}=p^{1-\varepsilon \Gamma^{*}},
$$

we find that

$$
p=\left[1-\frac{\Gamma^{*}}{\beta \varepsilon} \log \left(1+\varepsilon^{2} \beta z\right)\right]^{1 / \Gamma^{*}}+\varepsilon^{4} p_{g}(\mathbf{X}, T)+O\left(\varepsilon^{5}\right) .
$$

Here we have assumed the troposphere to be drier than the boundary layer and to have a potential temperature distribution

$$
\theta_{e}=1+\varepsilon^{2} \theta_{e}^{(2)}(z)+\cdots,
$$

where $\theta_{e}^{(2)}(z)=\beta z$ with a constant lapse rate $\beta$.

Asymptotic analysis also shows that the horizontal pressure gradient satisfies

$$
\begin{aligned}
\nabla_{X} p^{(i)} & =0 \quad \text { for } i=0,1,2,3 \text { and } \\
\nabla_{X} p^{(4)} & =-\left(\widehat{\Omega} \times \rho^{(0)} \mathbf{v}^{(0)}\right)_{\|},
\end{aligned}
$$

where $\rho^{(0)}=\exp (-z)$. As a consequence, the pressure term $p_{g}$ in (32) satisfies the geostrophic condition, that is,

$$
\nabla_{X} p_{g}=-\left(\widehat{\Omega} \times \rho^{(0)} \mathbf{v}^{(0)}\right)_{\|} .
$$


Because the pressure $p$ must be continuous at the top of the boundary layer as $z \rightarrow \delta^{3} H$, then $p \rightarrow p_{H}$, giving the required boundary condition for pressure. It follows that

$p_{H}=\left[1-\frac{\Gamma^{*}}{\beta \delta^{2}} \log \left(1+\delta^{7} \beta H\right)\right]^{1 / \Gamma^{*} \delta^{2}}+\delta^{8} p_{g}(\mathbf{X}, T)+O\left(\delta^{10}\right)$

which upon expansion in terms of $\delta$ implies that

$$
\begin{aligned}
p_{H}= & 1-\delta^{3} H+\frac{1}{2} \delta^{6} H^{2}-\frac{1}{2} \delta^{8} \Gamma^{*} H^{2}+\delta^{8} p_{g} \\
& -\frac{1}{6} \delta^{9} H^{3}+\frac{1}{2} \delta^{10} \beta H^{2} \cdots
\end{aligned}
$$

\section{2) VERTICAL MOMENTUM BALANCE AND EQUATION OF STATE EXPANSIONS}

Expanding the rescaled governing equation for the vertical momentum balance, and given the assumed velocity structure so that $w^{(i)}=0$ for $i<6$, implies that hydrostatic balances hold up to tenth order:

$$
p_{\eta}^{(i)}=0 \quad \text { for } \quad i=0,1,2
$$

and

$$
p_{\eta}^{(i)}+\rho^{(i-3)}=0 \text { for } i=3, \ldots, 10,
$$

where the first equation simply reflects our choice of expansion wherein $\rho^{(0)}$ is the leading-order term in the density. Integrating these equations over the depth of the boundary layer and combining with the boundary condition on $p_{H}$ and an expansion of the equation of state yields expressions for pressure that can be used to evaluate vertically integrated pressure gradients in terms of other known quantities. The derivation is presented in appendix B, leading to the following expressions for the bulk averaged pressure gradient:

$$
\begin{aligned}
& \left(\int_{0}^{H} \nabla_{X} p d \eta\right)^{(i)}=0 \text { for } i=0,1,2,3,4,5,6, \\
& \left(\int_{0}^{H} \nabla_{X} p d \eta\right)^{(7)}=\varphi^{(3)} H^{(0)} \nabla_{X} H^{(1)},
\end{aligned}
$$$$
\left(\int_{0}^{H} \nabla_{X} p d \eta\right)^{(8)}=H^{(0)} \nabla_{X} p_{g}+\varphi^{(3)}\left(H^{(0)} \nabla_{X} H^{(2)}\right.
$$$$
\left.+H^{(1)} \nabla_{X} H^{(1)}\right)+\varphi^{(4)} H^{(0)} \nabla_{X} H^{(1)},
$$

and

$$
\begin{aligned}
\left(\int_{0}^{H} \nabla_{X} p d \eta\right)^{(9)}= & H^{(1)} \nabla_{X} p_{g}+\varphi^{(3)}\left(H^{(0)} \nabla_{X} H^{(3)}+H^{(1)} \nabla_{X} H^{(2)}+H^{(0)} \nabla_{X} H^{(3)}\right)+\varphi^{(4)}\left(H^{(0)} \nabla_{X} H^{(2)}+H^{(1)} \nabla_{X} H^{(1)}\right) \\
& +\varphi^{(5)} H^{(0)} \nabla_{X} H^{(1)}+\frac{H^{(0)}}{2} \nabla_{X}\left(q_{t}^{(6)}-\theta_{e}^{(6)}\right)+\frac{\beta_{1}}{6}\left(\Gamma^{*} L_{v}^{*}-R^{*}\right) \nabla_{X} \eta_{c}^{3}
\end{aligned}
$$

where $\eta_{c}$ is the cloud-base height and $\varphi^{(i)}=\varphi^{(i)}\left(\theta_{e}^{(i)}, q_{t}^{(i)}\right.$, $\left.q_{v}^{(i)}\right)$ is given by (B8).

\section{e. Intermediate summary of the asymptotic equations}

At this point it proves useful to summarize the equations that we have derived on the basis of the analysis of this section. They are

\section{1) Mass BALANCE}

$$
\bar{E}^{(i)}=0 \quad \text { for } \quad i=0,1,2,3,4,5
$$

$\frac{\partial H^{(1)}}{\partial T}+H^{(0)} \nabla_{X} \cdot \mathbf{v}_{\|}^{(1)}+H^{(1)} \nabla_{X} \cdot \mathbf{v}_{\|}^{(0)}+\mathbf{v}_{\|}^{(0)} \nabla_{X} H^{(1)}=\bar{E}^{(6)}$.

\section{2) Horizontal MOMENTUM BALANCE}

$$
\begin{aligned}
& 0=\left(\overline{w \rho \mathbf{v}_{\|}}\right)_{0}^{(i)}+\left[\overline{\Delta\left(\rho \mathbf{v}_{\|}\right)_{H} E}\right]^{(i)} \text { for } \quad i=0,1,2,3, \\
& 0=\left(\overline{w \rho \mathbf{v}_{\|}}\right)_{0}^{(4)}+\left[\overline{\Delta\left(\rho \mathbf{v}_{\|}\right)_{H} E}\right]^{(4)}-\varphi^{(3)} H^{(0)} \nabla_{X} H^{(1)},
\end{aligned}
$$

and

$$
\begin{gathered}
H^{(0)}\left(\widehat{\Omega} \times \mathbf{v}^{(0)}\right)_{\|}+H^{(0)} \nabla_{X} p_{g}=\left(\overline{w \rho \mathbf{v}_{\|}}\right)_{0}^{(5)}+\left(\overline{\Delta\left(\rho \mathbf{v}_{\|}\right)_{H} E}\right]^{(5)} \\
-\varphi^{(4)} H^{(0)} \nabla_{X} H^{(1)}-\varphi^{(3)}\left(H^{(0)} \nabla_{X} H^{(2)}+H^{(1)} \nabla_{X} H^{(1)}\right) .
\end{gathered}
$$

The last two sets of equations indicate that the pressure gradients due to depth fluctuations are in balance with the surface momentum and entrainment fluxes [related to the jump $\Delta\left(\rho \mathbf{v}_{\|}\right)$at $\left.\eta=H\right]$, in particular fluxes $O\left(\delta^{4}\right)$ 
and $O\left(\delta^{5}\right)$. The implications of these fluxes on the flow are discussed in the next section. At the next order, we find an evolution equation for the first-order velocity perturbation,

$$
\begin{aligned}
H^{(0)} \frac{\partial \mathbf{v}_{\|}^{(1)}}{\partial T}+H^{(0)} \mathbf{v}_{\|}^{(0)} \cdot \nabla_{X} \mathbf{v}_{\|}^{(1)}+H^{(0)}\left(\widehat{\Omega} \times \mathbf{v}^{(1)}\right)_{\|}+\nabla_{X} \Phi= & \left(\overline{w \rho \mathbf{v}_{\|}}\right)_{0}^{(6)}+\left[\overline{\Delta\left(\rho \mathbf{v}_{\|}\right)_{H} E}\right]^{(6)}-\varphi^{(5)} H^{(0)} \nabla_{X} H^{(1)} \\
& -\varphi^{(4)}\left(H^{(1)} \nabla_{X} H^{(1)}+H^{(0)} \nabla_{X} H^{(2)}\right)-\varphi^{(3)}\left(H^{(0)} \nabla_{X} H^{(3)}\right. \\
& \left.+H^{(1)} \nabla_{X} H^{(2)}+H^{(2)} \nabla_{X} H^{(1)}\right)-H^{(1)}\left(\widehat{\Omega} \times \mathbf{v}^{(0)}\right)_{\|} \\
& -H^{(1)} \nabla_{X} p_{g},
\end{aligned}
$$

where

$$
\Phi=\frac{H^{(0)^{2}}}{2}\left(-\theta_{e}^{(6)}+q_{t}^{(6)}\right)+\frac{\beta_{1}}{6}\left(\Gamma^{*} L_{v}^{*}-R^{*}\right) \eta_{c}^{3} .
$$

The coupling between the thermodynamics and the momentum variables occurs through the (hydrostatic) pressure gradient. Nonlinearities in the momentum equation arise solely through this coupling as a result.

3) Equivalent Potential temperature $0=\left(\overline{w \rho \theta_{e}}\right)_{0}^{(i)}+\left(\overline{H\left\langle\rho \mathcal{S}_{\theta_{e}}\right\rangle}\right)^{(i-3)}$ for $i=0,1,2,3,4,5$,

$$
0=\left(\overline{w \rho \theta_{e}}\right)_{0}^{(i)}+\left[\overline{\Delta\left(\rho \theta_{e}\right)_{H} E}\right]^{(i)}+\left(\overline{H\left\langle\rho \mathcal{S}_{\theta_{e}}\right\rangle}\right)^{(i-3)}
$$$$
\text { for } i=6,7,8,9,10 \text {, }
$$

and

$$
\begin{aligned}
\frac{\partial \theta_{e}^{(6)}}{\partial T}+\mathbf{v}_{\|}^{(0)} \cdot \nabla_{X} \theta_{e}^{(6)=}= & \frac{\left(\overline{w \rho \theta_{e}}\right)_{0}^{(11)}}{H^{(0)}}+\frac{\left[\overline{\Delta\left(\rho \theta_{e}\right)_{H} E}\right]^{(11)}}{H^{(0)}} \\
& +\frac{\left(\overline{H\left\langle\rho \mathcal{S}_{\theta_{e}}\right\rangle}\right)^{(8)}}{H^{(0)}}
\end{aligned}
$$

\section{4) Total moisture CONTENT}

$$
0=\left(\overline{w \rho q_{t}}\right)_{0}^{(i)}+\left(\overline{H\left\langle\rho \mathcal{S}_{q_{t}}\right\rangle}\right)^{(i-3)} \quad \text { for } \quad i=0,1,2,3,4,5,
$$

$$
\begin{aligned}
& 0=\left(\overline{w \rho q_{t}}\right)_{0}^{(i)}+\left[\overline{\Delta\left(\rho q_{t}\right)_{H} E}\right]^{(i)}+\left(\overline{H\left\langle\rho \mathcal{S}_{q_{t}}\right\rangle}\right)^{(i-3)} \\
& \text { for } i=6,7,8,9,10
\end{aligned}
$$

and

$$
\begin{aligned}
\frac{\partial q_{t}^{(6)}}{\partial T}+\mathbf{v}_{\|}^{(0)} \cdot \nabla_{X} q_{t}^{(6)}= & \frac{\left(\overline{w \rho q_{t}}\right)_{0}^{(11)}}{H^{(0)}}+\frac{\left[\overline{\Delta\left(\rho q_{t}\right)_{H} E}\right]^{(11)}}{H^{(0)}} \\
& +\frac{\left(\overline{H\left\langle\rho \mathcal{S}_{q_{t}}\right.}\right)^{(8)}}{H^{(0)}}
\end{aligned}
$$

General remark: To separate asymptotic expansions from the additional scaling assumptions associated with specific parameterizations that are introduced in section 5 , we have carried all the orders formally expressing all physical quantities in terms of a minimal set of fundamental reference quantities. Depending on the specific perturbation regime, say, for the strength of the entrainment flux, the expansions then include a number of trivial lower-order terms with the physically relevant expressions appearing formally at very high (e.g., 11th) order. Once a specific regime is determined, it would be possible to rephrase our expansions in such a way that the perturbation equations would touch upon only the leading one to three terms in the expansions. For example, we have used $u_{\text {ref }}$ to scale both the horizontal and vertical components of velocity, whereas the vertical velocity $w \sim \delta^{6} u_{\text {ref. }}$. Since the nontrivial equivalent potential temperature for a weak jump is $\theta_{e} \sim \delta^{5} \theta_{\text {ref }}$, the entrainment flux would have appeared at the first order rather than at the 11th order had we rescaled $w$ and $\theta_{e}$ accordingly. The main motivation for including all the terms in the expansions was to ease further developments for different asymptotic limit regimes.

\section{Closure terms}

The equations described in the previous section include a variety of aggregated quantities that must be modeled or parameterized. These include surface and entrainment fluxes, radiative transfer, and precipitation processes. In the context of the distinguished limits we propose here, both the form of the model that we assume and the magnitude of the parameters each model introduces emerge as structural uncertainties as they contribute to the form of the leading-order equations that we identify. Most of the models introduced either arise from, or are modified by, the presence of the cloud layer. As such, the depth of this layer, or equivalently the liquid water path (LWP), emerges as an important parameter. Hence, in proposing models to close our equations it is also necessary to develop consistent asymptotic 
relations for the input required by such models. In this section we propose asymptotically consistent parameterizations for the radiative, surface, and entrainment fluxes, but first we present the prerequisite asymptotic analysis for the depth of the cloud layer.

\section{a. Liquid water asymptotics}

The liquid water mixing ratio is given by

$$
q_{l}= \begin{cases}q_{t}-q_{s}, & \text { if } q_{t}>q_{s} \\ 0, & \text { otherwise. }\end{cases}
$$

Hence an asymptotic representation of $q_{l}$ depends on the asymptotic representation of the saturation mixing ratio of water vapor $q_{s}$. By definition

$$
q_{s}(T)=\frac{1}{R^{*}} \frac{p_{s}^{\prime}(T)}{p_{d}^{\prime}}
$$

in which $T$ is the temperature, $p_{s}$ the saturation vapor pressure, $p_{d}$ is the partial pressure of dry air, and $R^{*}$ is the ratio of the gas constant as represented by the distinguished limit as in (10). Note that the total pressure is simply the sum of the partial pressures, and that here primes represent dimensional quantities. The saturation vapor pressure can be approximated as a function of temperature, for instance by integrating the ClausiusClapeyron equation about a reference temperature and vapor pressure. Doing so yields the following expression for the dimensionless saturation vapor mixing ratio:

$$
q_{s}=\frac{\delta^{3} p_{s}^{*} \exp \left[\frac{A^{*}}{\delta^{2}}\left(1-\frac{1}{T}\right)\right]}{R^{*} p-\delta^{3} R^{*} p_{s}^{*} \exp \left[\frac{A^{*}}{\delta^{2}}\left(1-\frac{1}{T}\right)\right]} .
$$

To arrive at this equation we have introduced the distinguished limit, $p_{s, \text { ref }}^{\prime} / p_{\text {ref }}=0.035 \approx \delta^{3} p_{s}^{*}$, and made use of the previous distinguished limits given by (10). Given the expansion for pressure, it follows that (58) can be written as an asymptotic series in $\delta$ as follows:

$$
\begin{aligned}
q_{s}= & \delta^{3} \frac{p_{s}^{*}}{R^{*}}\left[1+\delta A^{*} T^{(3)}+\delta^{2} A^{*}\left(T^{(4)}+\frac{1}{2} A^{*} T^{(3)^{2}}\right)\right] \\
& +\delta^{6}\left(\beta_{0}+\beta_{1} \eta\right)+O\left(\delta^{7}\right),
\end{aligned}
$$

where

$$
\begin{aligned}
\beta_{0} & =\frac{p_{s}^{*}}{R^{*}} A^{*}\left(T^{(5)}+A^{*} T^{(3)} T^{(4)}+\frac{1}{6} A^{* 2} T^{(3)^{3}}+\frac{p_{s}^{*}}{A^{*}}\right) \\
& \left.\equiv q_{s}^{(6)}\right|_{\eta=0}
\end{aligned}
$$

is the saturation mixing ratio at the surface and

$$
\beta_{1}=-\frac{p_{s}^{*}}{R^{*}}
$$

The expansion (59) with $T^{(3)}, T^{(4)}$, and $T^{(5)}$, given in appendix A by (A19), defines the component terms $q_{s}^{(4)}, q_{s}^{(5)}$, and $q_{s}^{(6)}$ implicitly.

To obtain an asymptotic expression for the cloud-base height $\eta_{c}$ we assume that the cloud base appears where the saturation mixing ratio matches the total mixing ratio in the subcloud layer; that is, $q_{t}\left(\eta_{c}\right)=q_{s}\left(\eta_{c}\right)$. Above the cloud base, we assume that all vapor in excess of saturation condenses to liquid water; that is, the total mixing ratio is given by $q_{t}=q_{s}+q_{l}$, which is the sum of the saturation mixing ratio $q_{s}$ and the liquid water mixing ratio $q_{l}$. Therefore,

$$
q_{t}^{(6)}\left(\eta_{c}\right)=q_{s}^{(6)}\left(\eta_{c}\right)=\beta_{0}+\beta_{1} \eta_{c}=q_{s, 0}^{(6)}+\beta_{1} \eta_{c}
$$

(recalling subscript 0 denotes values valid at the surface). Because $q_{t}^{(6)}$ is assumed to be independent of height (26b), we find that to leading order the cloud-base height is given by

$$
\eta_{c}=\frac{1}{\beta_{1}}\left(q_{t}^{(6)}-q_{s, 0}^{(6)}\right)
$$

These expressions can now be used with the definition of the liquid water content (56) to derive an expression for the depth-averaged liquid water path:

$$
\begin{aligned}
\left\langle q_{l}\right\rangle H & =\int_{\eta_{c}}^{H}\left(q_{t}-q_{s}\right) d \eta \\
& =\delta^{6}\left[\left(q_{t}^{(6)}-q_{s, 0}^{(6)}\right)\left(H-\eta_{c}\right)-\frac{\beta_{1}}{2}\left(H^{2}-\eta_{c}^{2}\right)\right]+\cdots \\
& =-\delta^{6} \frac{\beta_{1}}{2}\left(H-\eta_{c}\right)^{2}+\cdots
\end{aligned}
$$

Thus,

$$
\left\langle q_{l}^{(6)}\right\rangle H^{(0)}=-\frac{\beta_{1}}{2}\left(H^{(0)}-\eta_{c}\right)^{2},
$$

which shows, as expected, that the leading-order vertically averaged liquid water mixing ratio is proportional to the square of the cloud thickness.

\section{b. Radiative flux $\mathcal{S}_{\theta_{e}}$}

The source term that appears in the equivalent potential temperature balance is due to both longwave and shortwave radiative effects. In our analysis we assume a nocturnal situation for which only longwave fluxes are important. The upward and downward radiative fluxes are given by the expression 


$$
F_{L}^{\uparrow \downarrow}(z)=(1-\varepsilon) F_{\text {bnd }}^{\uparrow \downarrow}+\epsilon T^{4}
$$

which we have made dimensionless through the reference value of $\sigma T_{\text {ref }}^{4}=460 \mathrm{~W} \mathrm{~m}^{-2}$ with the StefanBoltzmann constant $\sigma=5.67 \times 10^{-8} \mathrm{~W} \mathrm{~m}^{-2} \mathrm{~K}^{-4}$. In (66) $F_{\text {bnd }}$ denotes the boundary longwave flux, taken as just above cloud top for the downward fluxes and just below cloud base for the upward fluxes: that is, $F_{L}(H)$ and $F_{L}\left(\eta_{c}\right)$, respectively. The emissivity, denoted by $\epsilon$, is taken to be independent of the direction of the radiances. It is parameterized following the suggestion of Stevens et al. (2005) as follows:

$$
\epsilon=1-\exp \left(-\delta^{-5} a^{*} \int_{\eta_{c}}^{\eta} \rho q_{l} d \eta\right) .
$$

The term in the exponent measures the extinction cross section of the liquid water, $a$, multiplied by the liquid water path. The parameterization of radiation hence introduces a further distinguished limit, namely that $a=$ $\varepsilon^{-5 / 2} a^{*}$.

The net longwave radiation flux is given by $F_{L}=F_{L}^{\uparrow}-$ $F_{L} \downarrow$ and expansions $O(\delta)$ lead to

$$
F^{(0)}=F_{L}^{\uparrow(0)}\left(\eta_{c}\right)-F_{L}^{\downarrow(0)}(H) \Rightarrow \frac{\partial F^{(0)}}{\partial \eta}=0 .
$$

Thus, to first approximation the radiative flux is constant. At next order we have the balance

$$
F^{(1)}=\Delta F^{(1)}\left(1-a^{*} \int_{\eta_{c}}^{\eta} q_{l}^{(6)} d \eta\right)
$$

where $\Delta F^{(1)} \equiv F_{L}^{\uparrow(1)}\left(\eta_{c}\right)-F_{L}^{\downarrow(1)}(H)$ defines the flux difference in the boundary fluxes. Therefore,

$$
\frac{\partial F^{(1)}}{\partial \eta}=-\Delta F^{(1)} a^{*} q_{l}^{(6)} .
$$

This expression shows that the radiative flux is responsive to changes in the modeled cloud structure through the liquid water.

Radiative flux divergences at first order influence the $\theta_{e}$ budget at a much lower order. This is evident from the dimensionless form of the equation for the equivalent potential temperature:

$$
\begin{aligned}
\frac{D}{D t}\left(\rho \theta_{e}\right) & =\rho \mathcal{S}_{\theta_{e}}=-\frac{\sigma T_{\text {ref }}^{4}}{c_{p} U_{\text {ref }} \rho_{\text {ref }} \theta_{\text {ref }}} \frac{\partial F}{\partial z}=-\delta^{10} \frac{\partial F}{\partial z} \\
& =-\delta^{7} \frac{\partial F}{\partial \eta}
\end{aligned}
$$

which, given that terms of order $F^{(1)}$ are the leading order in the forcing, implies that the following holds up to $i=8$ :

$$
\left(\rho \mathcal{S}_{\theta_{e}}\right)^{(i)}=0 \text { for } \quad i=0, \ldots, 7
$$

and

$$
\left(\rho \mathcal{S}_{\theta_{e}}\right)^{(8)}=-\frac{\partial F^{(1)}}{\partial \eta}=\Delta F^{(1)} a^{*} q_{l}^{(6)} .
$$

The depth-averaged source terms are thus given by

$$
\left\langle\mathcal{S}_{\theta_{e}}^{(i)}\right\rangle=0 \quad \text { for } \quad i=0,1,2,3,4,5,6,7,
$$

and

$$
\left\langle\mathcal{S}_{\theta_{e}}^{(8)}\right\rangle H^{(0)}=\Delta F^{(1)} a^{*}\left\langle q_{l}^{(6)}\right\rangle H^{(0)}=-\frac{\beta_{1}}{2} \Delta F^{(1)} a^{*}\left(H^{(0)}-\eta_{c}\right)^{2} .
$$

The asymptotics show that the radiative fluxes are related to the thickness of the cloud and are interactive in the sense the fluxes evolves, through its dependence on $\eta_{c}$, with the total water mixing ratio $q_{t}^{(6)}$ and the equivalent potential temperature $\theta_{e}^{(6)}$.

\section{c. Precipitation flux $\mathcal{S}_{q_{t}}$}

Formation of drizzle in cloudy air is an important mechanism for depleting cloud water. Thus, when there is drizzle, the total water is no longer a conserved quantity because of the reduction of the liquid water in the cloud layer and evaporation of the precipitation in the subcloud layer; thus, total water mixing ratio balance is

$$
\frac{D}{D t} \rho q_{t}=\rho \mathcal{S}_{q_{t}}=\rho R_{e}-\rho R_{p}
$$

where $R_{p}$ is the rate of production of precipitation and $R_{e}$ is the rate of evaporation of precipitation.

A simple bulk parameterization of $R_{p}$ of the form $R_{p}=C_{R} q_{l}^{\alpha_{p}}$, that is, the precipitation rate is parameterized as some fraction of the liquid water (with $C_{R}$ a precipitation conversion rate), has been proposed on the basis of a number of studies (Pawlowska and Brenguier 2003; Comstock et al. 2005; van Zanten et al. 2005). Although the evaporation of precipitation below stratocumulus can be substantial, it is neglected for reasons of simplicity in the present analysis. To the extent that the equations developed here demonstrate practical utility in understanding the interactions between the dynamics and thermodynamics of stratocumulus on the 
mesoscale, such effects (among others, e.g., shortwave radiation) can always be reintroduced parametrically.

Based on the aforementioned simple parameterization, the drizzle effect is included in the total water mixing ratio balance as

$$
\left\langle\mathcal{S}_{q_{t}}^{(8)}\right\rangle H^{(0)}=-\mathcal{P}^{*}\left\langle q_{l}^{(6)}\right\rangle^{\alpha_{p}} H^{(0)}=\frac{\beta_{1}}{2} \mathcal{P}^{*}\left(H^{(0)}-\eta_{c}\right)^{2 \alpha_{p}},
$$

where $\mathcal{P}^{*}$ is a constant $O(1)$ representing the precipitation conversion rate. In principle the flux of precipitation acts as a source of $\theta_{e}$ through the change to $q_{t}$ in that equation. These effects, however, will only appear at higher orders and, thus, do not enter into the asymptotic balances we explore.

\section{d. Parameterization of the surface fluxes}

To the extent that ocean currents are negligible the lower boundary conditions for the velocity components are zero (i.e., $\mathbf{v}_{\|}=0$ ). Other surface quantities are denoted, as before, by as subscript zero, so that the surface temperature is denoted by $T_{0}$. The equivalent potential temperature at the surface $\theta_{e, 0}=\theta_{e}\left(T_{0}, p_{s, 0}\right)$ as for a water-covered surface $q_{v, 0}=q_{s, 0}=q_{s}\left(T_{0}, p_{s, 0}\right)$. Hence, using the equation of state to express $\theta_{e, 0}^{(i)}$ in terms of $T_{0}^{(i)}$ and $q_{s, 0}^{(i)}$, it is straightforward to show that (58) becomes

$$
\begin{aligned}
q_{s, 0}= & \delta^{3} \frac{p_{s}^{*}}{R^{*}}\left[1+\delta A^{*} T_{0}^{(3)}+\delta^{2} A^{*}\left(T_{0}^{(4)}+A^{*} T_{0}^{(3)^{2}}\right)\right] \\
& +\delta^{6} \frac{p_{s}^{*}}{R^{*}} A^{*}\left(\frac{p_{s}^{*}}{A^{*}}+T_{0}^{(5)}+A^{*} T_{0}^{(3)} T_{0}^{(4)}+\frac{1}{6} A^{* 2} T_{0}^{(3)^{3}}\right) \\
& +O\left(\delta^{7}\right) .
\end{aligned}
$$

The parameterization of the surface fluxes is achieved by the use of the bulk transfer formulas

$$
\begin{aligned}
& \left(\overline{\rho \mathbf{v}_{\|} w}\right)_{0}=-C_{D} \rho_{0}\left|\mathbf{v}_{\|}\right| \mathbf{v}_{\|}, \\
& \left(\overline{\rho w \theta_{e}}\right)_{0}=-C_{H} \rho_{0}\left|\mathbf{v}_{\|}\right|\left(\theta_{e}-\theta_{e, 0}\right),
\end{aligned}
$$

and

$$
\left(\overline{\rho w q_{t}}\right)_{0}=-C_{Q} \rho_{0} \mid \mathbf{v}_{\| \mid}\left(q_{t}-q_{s, 0}\right),
$$

where $\left|\mathbf{v}_{\|}\right|=\sqrt{u^{2}+v^{2}}$ and the density at the surface $\rho_{0}=1+\delta^{3} \rho_{0}^{(3)}+\cdots$, as obtained from (B1)-(B7). The terms $C_{D}, C_{H}$, and $C_{Q}$ are the drag coefficients for momentum, sensible heat, and moisture, respectively. These are considered here to be constant, hence stability effects are not included in the surface exchange rules. The values of the exchange coefficients range from $1.4 \times 10^{-3}$ to $4 \times 10^{-3}$. We explore the weak flux limit wherein all coefficients are of the same order so that $C_{D, H, Q} \sim \delta^{6} C^{*}$ and $C^{*}$ is an $O(1)$ constant.

\section{1) Momentum fluxes}

For these limits the momentum fluxes take the form

$$
\begin{aligned}
(\overline{\rho u w})_{0}= & -C^{*} \delta^{6}\left|\mathbf{v}_{\|}\right|^{(0)} \mathbf{v}_{\|}^{(0)}-C^{*} \delta^{7}\left(\left|\mathbf{v}_{\|}\right|^{(1)} \mathbf{v}_{\|}^{(0)}\right. \\
& \left.+\left|\mathbf{v}_{\|}\right|^{(0)} \mathbf{v}_{\|}^{(1)}\right)+\cdots,
\end{aligned}
$$

where $\left|\mathbf{v}_{\|}\right|^{(0)}=\sqrt{u^{(0)^{2}+v^{(0)^{2}}}}$ and

$$
\left|\mathbf{v}_{\|}\right|^{(1)}=\frac{u^{(0)} v^{(1)}+u^{(1)} v^{(0)}}{\sqrt{u^{(0)^{2}}+v^{(0)^{2}}}} .
$$

Thus,

$$
\begin{aligned}
& \left(\overline{\rho \mathbf{v}_{\|} w}\right)_{0}^{(i)}=0 \quad \text { for } \quad i=0,1,2,3,4,5, \\
& (\overline{\rho u w})_{0}^{(6)}=-C^{*}\left|\mathbf{v}_{\|}\right|^{(0)} \mathbf{v}_{\|}^{(0)},
\end{aligned}
$$

and

$$
(\overline{\rho u w})_{0}^{(7)}=-C^{*}\left(\left|\mathbf{v}_{\|}\right|^{(0)} \mathbf{v}_{\|}^{(1)}+\left|\mathbf{v}_{\|}\right|^{(1)} \mathbf{v}_{\|}^{(0)}\right) .
$$

\section{2) Total MOISTURE FLUX}

In section 5c and (44) we found that $\mathcal{S}_{q_{t}}=\delta^{8} \mathcal{S}_{q_{t}}^{(8)}+\ldots$ and $E=\delta^{6} E^{(6)}+\cdots$; thus, it follows from (53) and (54) that

$$
\left(\overline{\rho w q_{t}}\right)_{0}^{(i)}=0 \quad \text { for } \quad i=0,1,2,3,4,5,6,7,8 .
$$

For a weak moisture jump

$$
\left(\overline{\rho w q_{t}}\right)_{0}^{(i)}=0 \quad \text { for } \quad i=9,10
$$

and a similar expansion as in the momentum flux gives

$$
\left(\overline{\rho w q_{t}}\right)_{0}^{(11)}=-C^{*}\left|\mathbf{v}_{\|}\right|^{(0)}\left(q_{t}^{(5)}-q_{s, 0}^{(5)}\right) .
$$

\section{3) EQuivalent POTENTIAL TEMPERATURE FLUX}

We found in section $5 \mathrm{~b}$ that $\mathcal{S}_{\theta_{e}} \sim \delta^{8}$; hence, given (51), the condition of sublinear growth and the assumption that entrainment effects first emerge for $i=11$ is consistent with 


$$
\left(\overline{\rho w \theta_{e}}\right)_{0}^{(i)}=0 \quad \text { for } \quad i=0,1,2,3,4,5,6,7,8,9,10 .
$$

This condition is consistent with our representation of surface fluxes, for which

$$
\left(\overline{\rho w \theta_{e}}\right)_{0}^{(11)}=-C^{*}\left|\mathbf{v}_{\|}\right|^{(0)}\left(\theta_{e}^{(5)}-\theta_{e, 0}^{(5)}\right) .
$$

\section{e. Entrainment velocity and entrainment flux}

The entrainment closure is usually based on the turbulent structure of the mixed layer. However, the lack of consensus on the entrainment rate identified by Stevens (2002) remains. In general, entrainment rates are expressed in terms of the surface heat flux into the boundary layer, cloud-top radiative flux out of the layer, radiative flux jump occurring inside the entrainment zone, and some assumption of entrainment buoyancy flux. In most studies the wind shear is usually neglected, although stratocumulus clouds simulations by Moeng (2000) show that an increase in shear leads to increase in entrainment rate by a significant amount. To avoid being tangled into the entrainment debate, we estimate the entrainment velocity based on results obtained from laboratory experiments that include stratification and shear effects.

Entrainment velocity $E$ as observed in mixing layer flow experiments with interfacial shear flows takes the form

$$
E=C_{E}|\Delta V| \mathrm{Ri}_{B}^{-n},
$$

where $\mathrm{Ri}_{B}$ is the bulk Richardson number based on the interfacial velocity jump $\Delta V$ defined as $\mathrm{Ri}_{B}=\Delta b H /$ $(\Delta V)^{2}$, where $H$ is the depth of the mixed layer and $\Delta b=g \Delta \rho / \rho$ is the buoyancy jump at the top of the layer. The parameter dependencies are similar to many of the entrainment laws that have been suggested in the literature (Stevens 2002), except that here we link mixing to the differences in the mean flow, as given by $\Delta V$ rather than a convective velocity scale. Laboratory experiments (e.g., Strang and Fernando 2001) identify three regimes with $n \approx 0$ for $\mathrm{Ri}_{B} \leq 1.5, n \approx 2.63 \pm 0.45$ for $1.5 \leq \mathrm{Ri}_{B} \leq 5$, and $n \approx 1.30 \pm 0.15$ for $5 \leq \mathrm{Ri}_{B} \leq 20$. Thus, for the depth $H \sim \delta^{3} h_{\mathrm{sc}}$, the weak stratification case $\Delta b \sim \delta^{5} g$, and the weak surface flux case $\Delta V \sim \delta v_{\text {ref }}$ we find that $\mathrm{Ri}_{B} \sim \delta^{6} g h_{\text {sc }} / v_{\text {ref }}^{2} \sim \delta^{6} \mathrm{Fr}^{-2} \sim \delta^{-2}$, making use of the distinguished limit (1). This value of the Richardson number falls under the third regime of Strang and Fernando's experiments. Therefore, the dimensionless entrainment velocity is given by

$$
E=C_{E}^{*} \delta^{2}|\Delta V|\left[\delta^{5} \frac{\rho(\Delta V)^{2}}{H \Delta \rho}\right]^{3 / 2}
$$

where we have taken the constant $C_{E}=0.02 \pm 0.01 \approx$ $\delta^{2} C_{E}^{*}$ and $n=1.5$.

The problem now reduces to finding an expression for interfacial density jump $\Delta \rho$. Recall that the pressure at the region above the boundary layer is given by (32); since in this region the pressure is hydrostatic, we find that

$$
\rho=-p_{z}=\frac{1}{\left(1+\varepsilon^{2} \beta z\right)}\left[1-\frac{\Gamma^{*}}{\beta \varepsilon} \log \left(1+\varepsilon^{2} \beta z\right)\right]^{\left(1 / \Gamma^{*} \varepsilon\right)-1}+O\left(\varepsilon^{5}\right),
$$

which implies that the density just above the inversion layer $\mathcal{Q}_{H+}$ is given by

$$
\rho_{H+}=1-\delta^{3} H+\delta^{5} \Gamma^{*} H+\frac{1}{2} \delta^{6} H^{2}-\delta^{7} \beta H+O\left(\delta^{8}\right) .
$$

From (B1) to (B7) we find that the density at the top of the layer is given by

$$
\begin{aligned}
\rho_{H-}= & 1-\delta^{3} H+\delta^{5}\left(\Gamma^{*} H+\varphi^{(5)}\right) \\
& +\delta^{6}\left(\frac{1}{2} H^{2}+\varphi^{(6)}\right)+O\left(\delta^{7}\right) .
\end{aligned}
$$

Therefore the density jump at the inversion layer is given by

$$
(\Delta \rho)_{H}=\rho_{H+}-\rho_{H-}=-\delta^{5} \varphi^{(5)}-\delta^{6} \varphi^{(6)}+O\left(\delta^{7}\right) .
$$

The velocity jump is given by $\Delta \mathbf{v}_{\|}=\mathbf{v}_{\| g}-\mathbf{v}_{\|}=\left(\mathbf{v}_{\| g}-\right.$ $\left.\mathbf{v}_{\|}^{(0)}\right)-\delta \mathbf{v}_{\|}^{(1)}+O\left(\delta^{2}\right)$ and, from $(48), \stackrel{\mathbf{v}_{\|}^{(0)}}{=}=\mathbf{v}_{\| g}$ for a weak surface momentum flux. This leads to a velocity jump $\left|\Delta \mathbf{v}_{\|}\right|=\delta\left|\mathbf{v}_{\|}^{(1)}\right|+O\left(\delta^{2}\right)$. Therefore, the entrainment velocity is given by

$$
E=\delta^{6} \frac{C_{E}^{*}\left(\mathbf{v}_{\|}^{(1)}\right)^{4}}{H^{(0)}\left(\varphi^{(5)}\right)^{3 / 2}}+O\left(\delta^{7}\right)
$$

where $\varphi^{(5)}$ given by (B8) is assumed nonnegative. Equation (97) states that the leading-order entrainment, $E^{(6)}$, depends mainly on $\mathbf{v}_{\|}^{(1)}$, which in turn depends on the thermodynamics variables $\theta^{(6)}$ and $q_{t}^{(6)}$ as per (49). The evolution of these variables, given by (52) and (55), depends on radiative flux and drizzle, respectively, in addition to the surface fluxes. Thus, we can conclude that entrainment rate given by (97) is based on the strength of the radiative driving of the layer and on contributions from other energetic sources (e.g., surface fluxes, wind shear at the boundary layer top, drizzle, etc.). 
For weak temperature jumps $\left(\Delta \theta_{e}\right)_{H}^{(5)}$ and weak moisture jumps $\left(\Delta q_{t}\right)_{H}^{(5)}$, the entrainment fluxes first appear at order 11; thus,

$$
\begin{aligned}
{\left[\overline{E \Delta\left(\rho \theta_{e}\right)_{H}}\right]^{(11)} } & =E^{(6)} \Delta\left(\rho \theta_{e}\right)_{H}^{(5)} \text { and }\left[\overline{E \Delta\left(\rho q_{t}\right)_{H}}\right]^{(11)} \\
& =E^{(6)} \Delta\left(\rho q_{t}\right)_{H}^{(5)} .
\end{aligned}
$$

\section{Weak interfacial buoyancy jump model}

\section{a. Summary of the closed systems of reduced equations}

The above discussion suggests that the structure of the resultant equations depend on the magnitude of temperature jump, moisture jump, and the drag coefficients. The case of weak interfacial stability is interesting because it allows for entrainment effects to emerge at lower orders and is discussed further below. For such a case the equivalent potential temperature has an asymptotic expansion of the form

$$
\begin{aligned}
\theta_{e}= & 1+\delta^{5} \theta_{e}^{(5)}+\delta^{6} \theta_{e}^{(6)}(\mathbf{X}, T)+\delta^{7} \theta_{e}^{(7)}(\mathbf{X}, \eta, T) \\
& +\delta^{8} \theta_{e}^{(8)}(\mathbf{X}, \xi, \eta, T, \tau)+\cdots
\end{aligned}
$$

The momentum balance Eq. (48) thus reduces to

$$
\left(\widehat{\boldsymbol{\Omega}} \times \mathbf{v}^{(0)}\right)_{\|}+\nabla_{X} p_{g}=0,
$$

and this places a constraint on the background flow $\mathbf{v}_{\|}^{(0)}$. Equation (100) also implies that $\nabla_{X} \cdot \mathbf{v}_{\|}^{(0)}=0$.

Given the above, the closure terms following the discussion in section 5 result in the following system of equations for the evolution of the intermediate or mesoscale boundary layer flows:

$$
{\frac{\partial H^{(1)}}{\partial T}}^{+\mathbf{v}_{\|}^{(0)}} \cdot \nabla_{X} H^{(1)}+H^{(0)} \nabla_{X} \cdot \mathbf{v}_{\|}^{(1)}-\frac{C_{E}^{*}\left(\mathbf{v}_{\|}^{(1)}\right)^{4}}{H^{(0)}\left(\varphi^{(5)}\right)^{3 / 2}}=0,
$$

$$
\begin{aligned}
& \frac{\partial \mathbf{v}_{\|}^{(1)}}{\partial T}+\mathbf{v}_{\|}^{(0)} \cdot \nabla_{X} \mathbf{v}_{\|}^{(1)}+\left(\widehat{\mathbf{\Omega}} \times \mathbf{v}^{(1)}\right)_{\|}+\varphi^{(5)} \nabla_{X} H^{(1)} \\
& +\frac{1}{H^{(0)}} \nabla_{X} \Phi+\frac{C^{*}}{H^{(0)}} \mid \mathbf{v}_{\|}{ }^{(0)} \mathbf{v}_{\|}^{(0)}=0, \\
& \frac{\partial \theta_{e}^{(6)}}{\partial T}+\mathbf{v}_{\|}^{(0)} \cdot \nabla_{X} \theta_{e}^{(6)}+\frac{\beta_{1} a^{*}}{2 H^{(0)}} \Delta F^{(1)}\left(H^{(0)}-\eta_{c}\right)^{2} \\
& -\frac{C_{E}^{*}\left(\mathbf{v}_{\|}^{(1)}\right)^{4} \Delta\left(\rho \theta_{e}\right)_{H}^{(5)}}{H^{(0)^{2}}\left(\varphi^{(5)}\right)^{3 / 2}}+\frac{C^{*}}{H^{(0)}}\left(\left|\mathbf{v}_{\|}\right|^{(0)}\left(\theta_{e}^{(5)}-\theta_{e, 0}^{(5)}\right)=0,\right.
\end{aligned}
$$

and

$$
\begin{aligned}
& \frac{\partial q_{t}^{(6)}}{\partial T}+\mathbf{v}_{\|}^{(0)} \cdot \nabla_{X} q_{t}^{(6)}-\frac{\beta_{1}}{2 H^{(0)}} \mathcal{P}^{*}\left(H^{(0)}-\eta_{c}\right)^{2 \alpha_{p}} \\
& -\frac{C_{E}^{*}\left(\mathbf{v}_{\|}^{(1)}\right)^{4} \Delta\left(\rho q_{t}\right)_{H}^{(5)}}{H^{(0)^{2}}\left(\varphi^{(5)}\right)^{3 / 2}}+\frac{C^{*}}{H^{(0)}} \mid \mathbf{v}_{\|}{ }^{(0)}\left(q_{t}^{(5)}-q_{s, 0}^{(5)}\right)=0,
\end{aligned}
$$

where

$$
\Phi=\frac{H^{(0)^{2}}}{2}\left(-\theta_{e}^{(6)}+q_{t}^{(6)}\right)+\frac{\beta_{1}}{6}\left(\Gamma^{*} L_{v}^{*}-R^{*}\right) \eta_{c}^{3},
$$

the cloud-base height $\eta_{c}$ is given by (63), and $\varphi^{(5)}=$ $-\theta_{e}^{(5)}+\left(1+\Gamma^{*} L_{v}^{*}-R^{*}\right) q_{t}^{(5)}$.

\section{b. Illustrative solutions}

\section{1) STEAdy-STATE SOLUTiOns}

We now consider some steady-state features of the model described by (101)-(104) to demonstrate that it gives physically realistic results. The steady-state results are obtained by neglecting the time derivatives in the equations; for simplicity, we assume a unidirectional flow (i.e., $\partial / \partial y=0$ ), the $v$-component wind is set to zero, and we also neglect the Coriolis force in the mixed layer. The background wind speed flow is assumed to be equal to the geostrophic wind above the mixed layer. We further neglect horizontal variation of moisture and momentum. In this case, (104) simply shows that the steady-state flow is a balance among the surface latent heat, entrainment moisture fluxes, and precipitation; therefore, the entrainment velocity is given by

$$
\mathcal{E} \bar{u}^{4}=\frac{\mathcal{C}^{*}\left|u^{(0)}\right| \Delta_{s} Q_{t}}{\Delta Q_{t}}-\frac{\beta_{1} \mathcal{P}^{*}}{2 \Delta Q_{t}}\left(H^{(0)}-\bar{\eta}_{c}\right)^{2 \alpha_{p}},
$$

where the velocity perturbation $\mathbf{v}_{\|}^{(1)}=\bar{u} \mathbf{i}$, the entrainment coefficient is denoted by $\mathcal{E}=C_{E}^{*} / H^{(0)} \varphi^{(5)^{-3 / 2}}, \bar{\eta}_{c}$ is the steady cloud-base height, $\Delta \Theta_{e}=-\theta_{e}^{(5)}$ is the cloudtop jump in equivalent potential temperature, and $\Delta Q_{t}=$ $-q_{t}^{(5)}$ is the cloud-top jump in total water mixing ratio, with $\Delta_{s} \Theta_{e}=\left(\theta_{e}^{(5)}-\theta_{e, 0}^{(5)}\right)$ the surface temperature jump, $\Delta_{s} Q_{t}=\left(q_{t}^{(5)}-q_{s, 0}^{(5)}\right)$ the surface moisture jump, and $\varphi^{(5)}=$ $\Delta \Theta_{e}-\left(1+\Gamma^{*} L_{v}^{*}-R^{*}\right) \Delta Q_{t}$ the buoyancy jump.

Making use of expression (105) together with (101)(103), it is found that the leading-order steady-state cloud thickness $\left(H^{(0)}-\bar{\eta}_{c}\right)$ is a solution of the polynomial equation

$$
\gamma_{1}\left(H^{(0)}-\bar{\eta}_{c}\right)^{2 \alpha_{p}}+\gamma_{2}\left(H^{(0)}-\bar{\eta}_{c}\right)^{2}+\gamma_{3}=0,
$$


with the coefficients

$$
\begin{aligned}
& \gamma_{1}=-\mathcal{P} * \beta_{1}\left(2 \varphi-\Delta \Theta_{e}\right), \\
& \gamma_{2}=a^{*} \beta_{1} \Delta F \Delta Q_{t},
\end{aligned}
$$

and

$$
\begin{aligned}
\gamma_{3}= & 2 C^{*} \mid u^{(0)}\left\{\Delta_{s} Q_{t}\left(2 \varphi-\Delta \theta_{e}\right)\right. \\
& \left.+\left[\frac{2}{H^{(0)}}\left(u^{(0)}\right)^{2}+\Delta_{s} \Theta_{e}\right] \Delta \Theta_{e}\right\} .
\end{aligned}
$$

The cloud layer is assumed only in cases where the root of polynomial equation (106) satisfies $0 \leq \bar{\eta}_{c} \leq H^{(0)}$.

Equations (105) and (106) are solved by specifying the values of the parameters, unless noted otherwise as given in Table 6 . These values are reasonably representative for the stratocumulus clouds [see, e.g., Stevens et al. (2005) for typical values]. We set the precipitation tuning parameter $\alpha_{p}$ to $3 / 2$ based on the results obtained by van Zanten et al. (2005). The standard precipitation formation rate is given by $\mathcal{P}^{*}=1$. A value greater than this will denote high precipitation efficiency, whereas a value less than one is considered to be low precipitation efficiency. The value $\mathcal{P}^{*}=0$ corresponds to switching off the precipitation.

We focus on equilibria as a function of the net radiative flux, the surface sensible heat flux, and surface latent heat flux for a given precipitation conversion coefficient $\mathcal{P}^{*}$. In this study we do not present any results on the effect of the depth of boundary layer and the background wind, since the main effect of reducing the depth of the boundary layer is in reducing the extent in parameter space where cloudy equilibria are found. The principal effect of changing the wind speed is to change the surface fluxes, with the larger $u^{(0)}$ corresponding to larger surface fluxes. For a fixed surface flux, this is equivalent to varying the bulk coefficients, with larger $u^{(0)}$ corresponding to smaller coefficients. Here the drag coefficient is held fixed at $C^{*}=$ 0.4, which corresponds to $C_{D}=C_{H}=C_{Q}=0.0014$.

The effect of these parameters on the entrainment rate and liquid water path are shown in Fig. 1 and are summarized as follows:

(i) The effect of variations in surface latent heat flux are evaluated by varying $\Delta_{s} Q_{t}$. The results shown in Figs. $1 \mathrm{a}$ and $1 \mathrm{~b}$ are consistent with the widely accepted view that the entrainment is strongly influenced by the surface moisture flux (Moeng 2000), with larger surface moisture fluxes driving more entrainment.

(ii) To see the effect of the precipitation, we computed the equilibrium solution with the drizzle conversion
TABLE 6. Standard values of parameters used in the simulations.

\begin{tabular}{ccc}
\hline \hline Parameter & Dimensionless value & Approximate value \\
\hline$H^{(0)}$ & 1.5 & $900 \mathrm{~m}$ \\
$u^{(0)}$ & 0.5 & $10 \mathrm{~m} \mathrm{~s}^{-1}$ \\
$\Delta F$ & 3 & $75 \mathrm{~W} \mathrm{~m}^{2}$ \\
$\Delta \Theta_{e}$ & 1 & $2.3 \mathrm{~K}$ \\
$\Delta Q_{t}$ & -1 & $8 \mathrm{~g} \mathrm{~kg}^{-1}$ \\
$\Delta_{s} \Theta_{e}$ & -1.1 & $15 \mathrm{~W} \mathrm{~m}^{-2}$ \\
$\Delta_{s} Q_{t}$ & -0.8 & $90 \mathrm{~W} \mathrm{~m}^{-2}$ \\
$\mathcal{P}^{*}$ & 1 & $10^{-4} \mathrm{~s}^{-1}$ \\
$\alpha_{p}$ & $3 / 2$ & \\
\hline
\end{tabular}

rate $\mathcal{P}^{*}=0,0.5,1,1.5,2$ and compared the behavior of the precipitation rate with the increase of the latent heat flux. These figures show the consistent behavior similar to that described in Lenderink and Siebesma (2004), namely that the effect of the drizzle is to reduce the entrainment rate and that the liquid water path is reduced by heavy drizzle (Stevens et al. 1998).

(iii) The effect of the radiative driving on the layer is shown in Figs. 1c and 1d. An increase in radiative cooling in the boundary layer leads to a marked increase in the entrainment velocity, which dries the layer and thins the clouds. This trend, which is exaggerated because the cloud thinning is not allowed to feed back on radiation in the present example, is also discussed in Moeng et al. (1999). In absence of the precipitation, the equilibrium boundary layer is driven by a balance between the surface moisture flux and the entrainment flux only. Thus, the entrainment levels off in the absence of precipitation for a fixed latent heat flux.

(iv) Figures 1e and 1f show that the net impact of surface sensible heat flux on the entrainment rate and cloud thickness are small for a given precipitation rate. The trend of the computed values is in good agreement with the mixed layer analysis of Pelly and Belcher (2001) and observations by Moeng (2000).

\section{2) LiNEAR STABILITY ANALYSIS}

A linear stability analysis of the steady-state solutions is carried out in terms of normal modes. The resulting linearized eigenvalue problems describing the evolution of perturbations and the dispersion relation, namely,

$$
\begin{aligned}
& \sigma^{4}+i \mathcal{C}_{6} \sigma^{3}+\left[i\left(\mathcal{C}_{7} \mathcal{C}_{3}+\mathcal{C}_{5} \mathcal{C}_{2}+\mathcal{C}_{1} \varphi^{(5)}\right) k-\varphi^{(5)} H^{(0)} k^{2}\right] \sigma^{2} \\
& +\left[\left(\mathcal{C}_{7} \mathcal{C}_{4} \mathcal{C}_{2}-\mathcal{C}_{6} \mathcal{C}_{5} \mathcal{C}_{2}-\mathcal{C}_{6} \mathcal{C}_{1} \varphi^{(5)}\right) k-\varphi^{(5)} H^{(0)} \mathcal{C}_{6} k^{2}\right] \sigma=0
\end{aligned}
$$


(a)

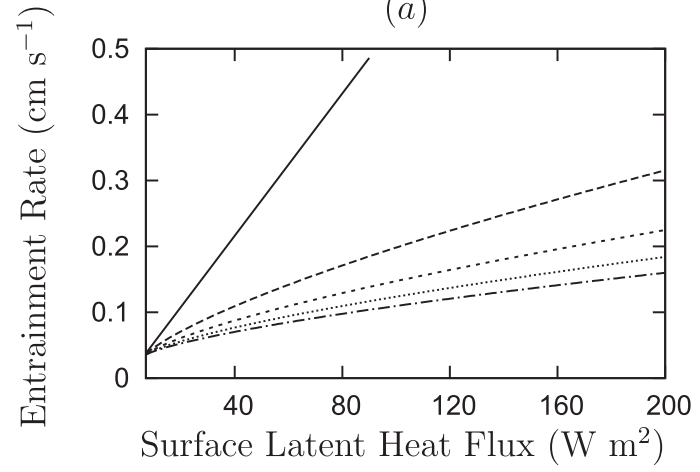

(c)

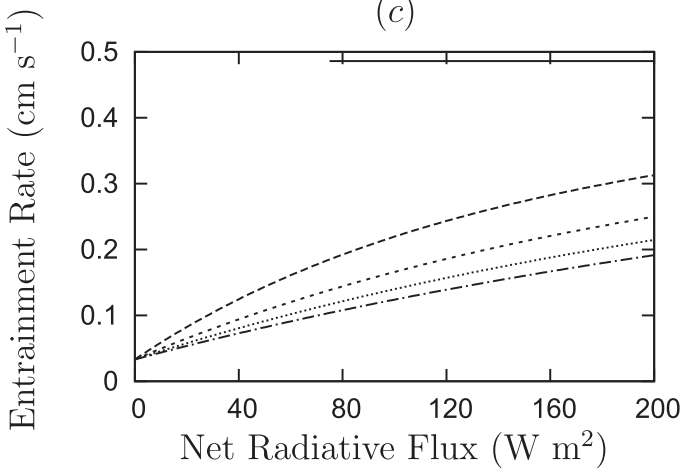

(e)

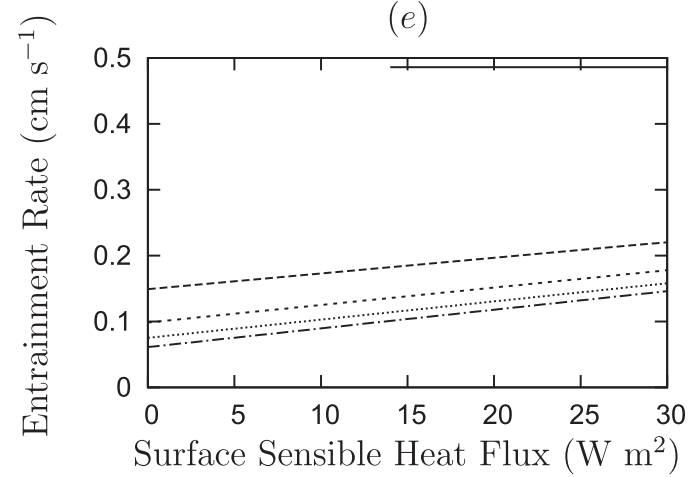

(b)

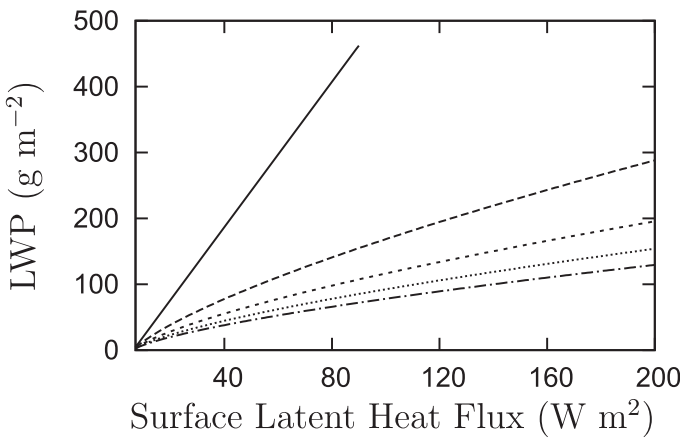

(d)

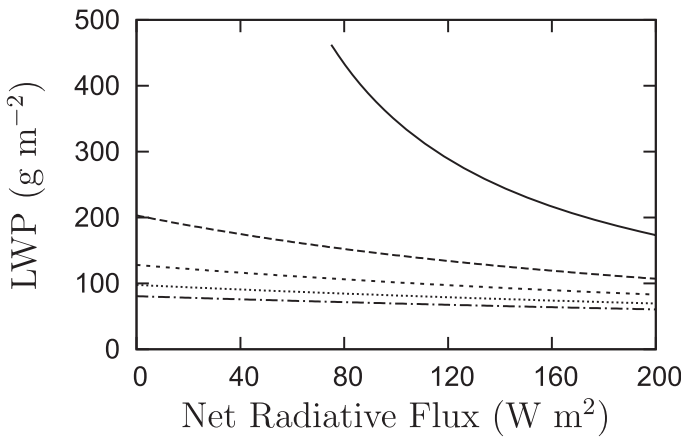

$(f)$

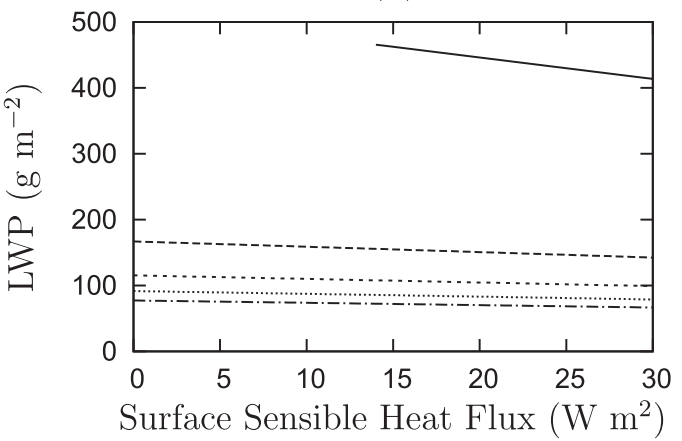

FIG. 1. The steady-state entrainment rate and liquid water path (LWP) as functions of the (a),(b) surface latent heat flux, (c),(d) net radiative flux, and (e),(f) surface sensible heat flux for various autoconversion efficiency rates: $\mathcal{P}^{*}=0$ (solid), 0.5 (dashed), 1.0 (short dashed), 1.5 (dotted), and 2.0 (dotted-dashed).

are derived in appendix C. The $C_{i}$ are expressions involving the model parameters as given in that appendix. The dispersion relation is a quartic equation for the intrinsic frequency $\sigma=\omega-u^{(0)} k$; thus, one finds four independent propagating modes at each wavenumber $k$. One of the modes is neutral and travels with the background wind. The imaginary part of the frequency $\omega$ represents the growth rate and the real part the temporal frequency. If the imaginary part of the frequency is positive, then the flow is unstable. In the following subsections, various properties of the other three modes are discussed in terms of the growth rates and phase velocities.

\section{(i) Wavenumber $k=0$}

In the long wavelength limit, the dispersion relation (107) reduces to

$$
\begin{aligned}
\omega^{4}+i \mathcal{C}_{6} \omega^{3} & =0 \Rightarrow \omega=0 ; \quad \omega=-i \mathcal{C}_{6} \\
& =-i \frac{\alpha_{p}}{H^{(0)}} \mathcal{P}^{*}\left(H^{(0)}-\bar{\eta}_{c}\right)^{\left(2 \alpha_{p}-1\right)},
\end{aligned}
$$

leading to three stationary neutral mode with $\omega=0$ and a stationary mode that decay at a rate of $\alpha_{p} \mathcal{P}^{*}\left(H^{(0)}\right.$ $\left.\bar{\eta}_{c}\right)^{\left(2 \alpha_{p}-1\right)} / H^{(0)}$. Thus, the steady-state solutions in the longwave limit are stable. The maximum damping rates 
(a)

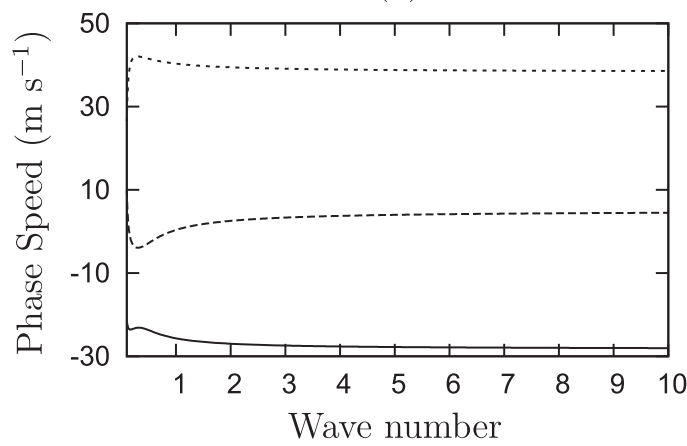

(b)

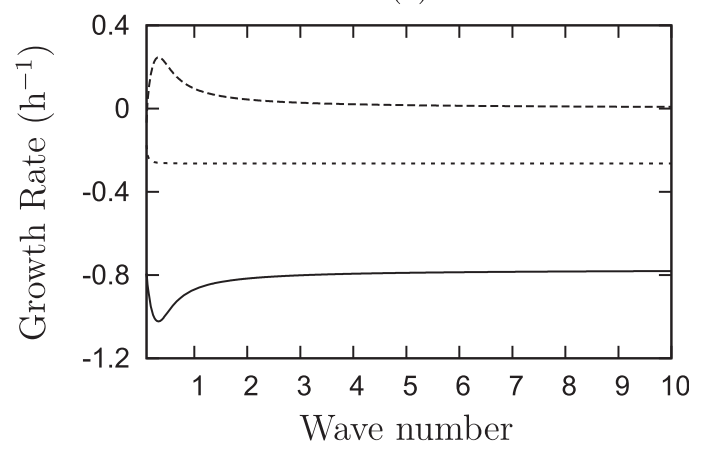

FIG. 2. (a) Phase speeds and (b) growth rates as functions of the wavenumbers for waves W1 (solid), W2 (dashed), and W3 (short dashed).

depends on the precipitation efficiency rate and the liquid water path. Thus, the damping rate increases with increase in the surface latent heat flux, decreases with increase in radiative flux, and marginally decreases with increase in surface sensible heat flux for a fixed precipitation rate.

\section{(ii) Wavenumber $k>0$}

We now illustrate the variations of the phase speed and growth rate of the other three nonneutral eigenmodes with wavenumbers in Fig. 2 for the standard values of the parameters given in Table 6 . The figure shows that generally the modes have phase speeds that approach $u^{(0)}-\sqrt{\varphi^{(5)} H^{(0)}}, u^{(0)}$, and $u^{(0)}+\sqrt{\varphi^{(5)} H^{(0)}}$ for sufficiently large wavenumber. We shall refer to these modes as W1, W2, and W3 respectively. The waves $\mathrm{W} 1$ and $\mathrm{W} 3$ capture the contribution of the background moisture states through $\varphi^{(5)}$ (an "effective gravity") and thus can be considered as moist gravity waves. The linear stability analysis reveals no scale-selective instability in any of the regimes considered. The figure shows that the system contains one unstable mode corresponding to $\mathrm{W} 2$ with maximum growth rate $0.25 \mathrm{~h}^{-1}$; it grows more slowly as the wavenumber increases. The phase speed corresponding to this maximum growth rate drops below the background wind to $-4 \mathrm{~m} \mathrm{~s}^{-1}$. Modes $\mathrm{W} 1$ and W3 are stable for the chosen parameters with maximum decay rate $0.25 \mathrm{~h}^{-1}$ and $0.8 \mathrm{~h}^{-1}$, respectively. The figure also shows that the system is only marginally unstable for wavenumber $k>1$.

We now investigate whether the structure of the waves $\mathrm{W} 1, \mathrm{~W} 2$, and $\mathrm{W} 3$ shows any mechanisms of instability as the parameter values change. The sensitivity of our model to parameter variations is shown in Fig. 3 for wavenumber 1 and 10. In general, the phase speed variation caused by changes in the precipitation efficiency rate, surface latent heat flux, radiative flux, and surface sensible heat flux are negligible, and thus we only describe the growth rates for the sensitivity experiments. Figure 3 shows that the stability of the W1 mode and the instability of the M3 are generally robust with the growth rate changing slightly when the precipitation efficiency rate, radiative flux, and sensible heat flux are varied. However, the decay rate of W1 increases significantly with the increase in the surface latent heat flux. The same applies to W3, as shown in Fig. 3b. The figures also reveal a parameter sensitivity to W3 that is different from W1 and W2-in particular, that there exists a critical precipitation efficiency rate below which mode W3 is unstable, as shown in Fig. 3a. Once the moist gravity wave (W3) instability disappears as the precipitation rate increases, the instability of the steadystate solution is replaced by an W2 instability whose growth rate is marginal. Finally, another aspect of W3 is that an increase in radiative flux surface and sensible heat flux leads to a decrease in decay rates (Figs. 3c,d).

\section{Concluding remarks}

In this paper we have derived sets of equations to explore the coupling between the thermodynamic state and the dynamic evolution of cloud-topped boundary layers on the mesoscale. Our analysis is the first to formally explore the multiscale asymptotics of flows in which moisture effects are captured through the use of moist conserved variables-and connected asymptotically through a distinguished limit-to the dynamic structure of the flow. Although our analysis focuses on a specific regime, the methods developed here may be of broader utility. At the very least they identify the difficulties moist atmospheric flows pose for a formal multiscale analysis.

Because our derivation formally relates approximations that one often makes in the representation of the 
(a)

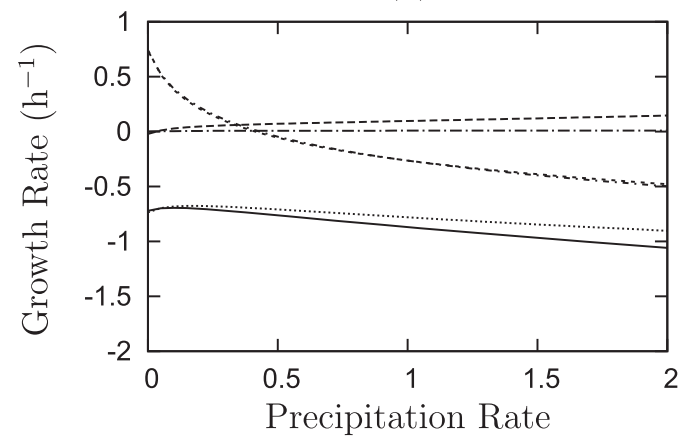

(c)

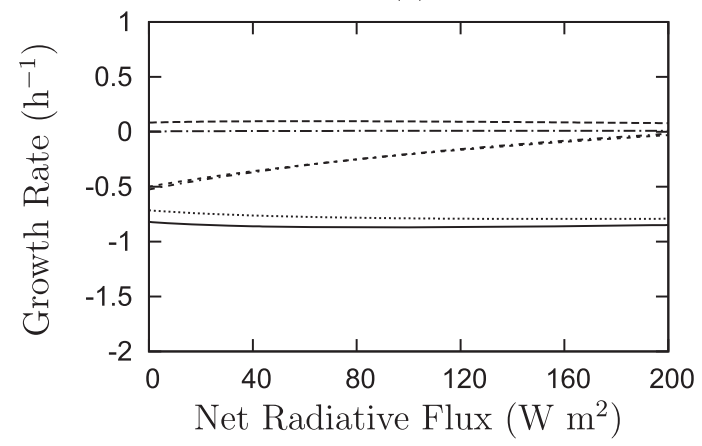

(b)

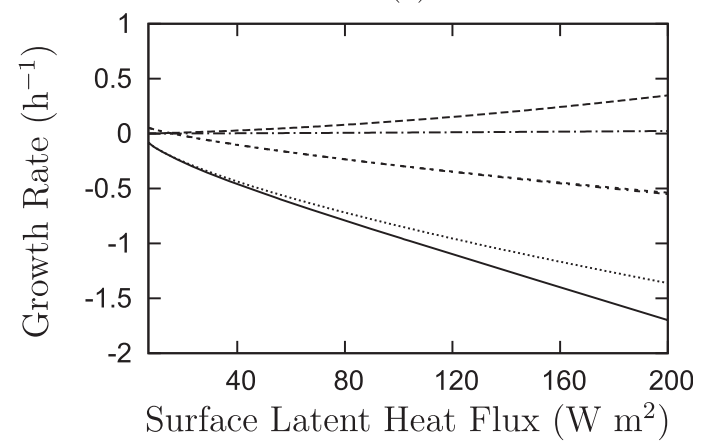

$(d)$

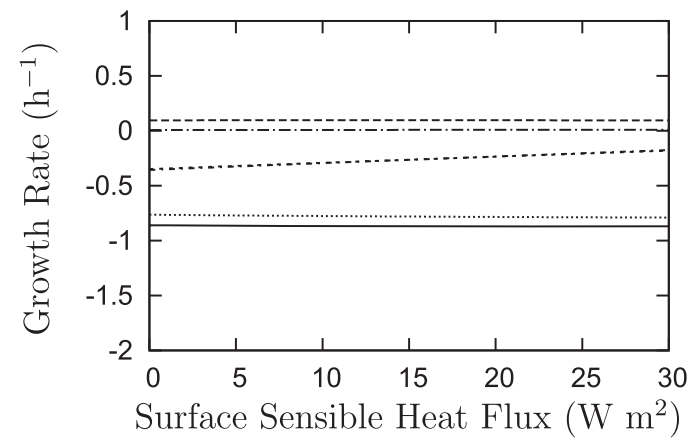

FIG. 3. Growth rates as functions of (a) the precipitation efficiency rate, (b) latent heat flux, (c) radiative flux, and (d) surface heat flux, for wavenumber 1, W1 (solid), W2 (dashed), and W3 (short dashed) and, for wavenumber 10, W1 (dotted), W2 (dotted-dashed) and W3 (dotted-short dashed).

thermodynamics properties of the fluid to simplifications one would like to achieve in the dynamics, it provides a formal justification for the bulk models frequently encountered in the literature. The equations developed here have filtered out the fast time scale and small spatial scales and identify a type of bulk model. In addition to the identification of a new reduced model for investigating the coupling of the fluid dynamics of the boundary layer on the mesoscale to the turbulent dynamics on the convective scale, a significant finding of this work is a strong link between perturbation velocity $\mathbf{v}_{\|}^{(1)}$ and the thermodynamics perturbations. Traditional bulk models ignore the coupling between the shallow-water-like dynamics of mesoscale motions and the fast turbulent dynamics that dominate the convective scales.

Further qualities of the reduced equations that we derive include

- the evolution of $\mathbf{v}_{\|}^{(1)}$ depends on the moist thermodynamics in addition to the depth of the boundary layer and surface fluxes of momentum;

- the entrainment rate depends directly on $\mathbf{v}_{\|}^{(1)}$, and through the evolution of this quantity on the accumulated effects of surface heat fluxes, the buoyancy jump across the inversion layer, radiative cooling, and any drizzle effects; and

- the velocity perturbations also driven by a depth perturbation dependent on stratification, which is $H^{(1)}$ for the case of weak stratification on which we focus.

It is obvious that much more could be done with the model than is reported here. We leave a more substantial investigation of the model's features to future work but note that the derived bulk equations give steady-state results that are physically realistic. For example, the trend of the computed steady-state entrainment rates agrees qualitatively with large-eddy simulations and observational studies, and the steadystate liquid water path also shows the general character of a precipitating cloud. We further showed that the introduction of precipitation may be important for the behavior of moist gravity waves. The linear stability analysis reveals that the stability of the steady-state solution does display some sensitivity to the parameter that determines the efficiency with which precipitation forms.

Acknowledgments. The authors thank Prof. Andrew Majda for constructive discussions. This work was 
supported by Deutsche Forschungsgemeinschaft (DFG) under Grant KL 611/14 and DFG Priority Research Program SPP 1167 "Quantitative Precipitation Forecast"; and by Leibniz-Gemeinschaft (WGL) through the PAKT Project "High-Resolution Modelling of Clouds and Gravity Waves". B.S. thanks Freie Universität Berlin for financing an extended initial visit in 2007 through the Interdisciplinary Center "Efficient Mathematical Modelling in the Earth and Life Sciences". The authors thank the two anonymous reviewers and the editor, K. K. Tung, for their useful comments and encouragement.

\section{APPENDIX A}

\section{Thermodynamic Relations}

\section{a. Equivalent potential temperature $\theta_{e}$}

The dimensional equivalent potential temperature is given by

$$
\theta_{e}^{\prime}=T^{\prime}\left(\frac{p_{00}}{p^{\prime}}\right)^{R_{d} / c_{p_{*}}} \Omega_{e} \exp \left(\frac{L_{v} q_{v}}{c_{p *} T^{\prime}}\right)
$$

where $p$ is the pressure, $p_{00}=p_{\text {ref }}=1000 \mathrm{hPa}$ is the reference pressure, $L_{V}$ is latent heat of vaporization, $T$ temperature; $q_{t}=q_{v}+q_{l}$ is the total water mixing ratio with $q_{v}$ the water vapor mixing ratio and $q_{l}$ the water content mixing ratio, and $c_{p *}=c_{p d}+c_{l} q_{t}$ in which $c_{p d}$ and $c_{l}$ are specific heat capacities for dry air and water, respectively. Now we have

$$
\frac{R_{d}}{c_{p *}}=\frac{R_{d}}{c_{p d}+c_{l} q_{t}}=\frac{R_{d}}{c_{p d}}\left(1+\frac{c_{l}}{c_{p d}} q_{t}\right)^{-1},
$$

where $R_{d}$ is the gas constants for dry air. We have

$$
\frac{R_{d}}{c_{p d}}=\frac{\gamma-1}{\gamma}=\frac{2}{7} \approx \Gamma^{*} \varepsilon, \quad \frac{c_{l}}{c_{p d}}=\frac{4217}{1007} \approx c_{p}^{*} \varepsilon^{-1}
$$

neglecting any variations of these values with temperature and pressure. The asterisk superscripts indicate constants of order one. Thus,

$$
\frac{R_{d}}{c_{p_{*}}}=\Gamma^{*} \varepsilon\left(1+c_{p}^{*} \varepsilon^{-1} q_{t}\right)^{-1}
$$

Also,

$$
\begin{aligned}
\frac{R_{v}}{c_{p_{*}}} & =\frac{R_{v}}{R_{d}} \frac{R_{d}}{c_{p *}}=R^{*} \Gamma^{*} \varepsilon\left(1+c_{p}^{*} \varepsilon^{-1} q_{t}\right)^{-1} \text { with } \\
\frac{R_{v}}{R_{d}} & =\frac{461.5}{287.0} \approx 1.61 \sim \varepsilon^{0} \equiv R^{*},
\end{aligned}
$$

where $R_{v}$ is the gas constant for water vapor.

The equation of state is given by

$$
p^{\prime}=\rho^{\prime} R_{d} T^{\prime}\left(1+q_{v} \frac{R_{v}}{R_{d}}\right)\left(1+q_{t}\right)^{-1},
$$

which implicitly defines expressions for the effective (two-phase) gas constant $R$ :

$$
R=R_{d}\left(1+q_{v} \frac{R_{v}}{R_{d}}\right)\left(1+q_{t}\right)^{-1}
$$

The dimensionless temperature follows as

$$
T=T^{\prime} / T_{\mathrm{ref}}=T^{\prime}\left(\frac{R_{d} \rho_{\mathrm{ref}}}{p_{\mathrm{ref}}}\right)=\frac{p}{\rho} \frac{\left(1+q_{t}\right)}{\left(1+R^{*} q_{v}\right)} .
$$

These relations can be used to define the dimensionless enthalpy ratio:

$$
\begin{aligned}
\frac{L_{v} q_{v}}{c_{p_{*}} T} & =\frac{L_{v} q_{v}}{c_{p_{*}}} \frac{\rho R_{d}}{p} \frac{\left(1+q_{v} R_{v} / R_{d}\right)}{\left(1+q_{t}\right)} \\
& \equiv L_{v}^{*} \Gamma^{*}\left(1+c_{p}^{*} \varepsilon^{-1} q_{t}\right)^{-1} \frac{\rho q_{v}}{p} \frac{\left(1+R^{*} q_{v}\right)}{\left(1+q_{t}\right)}
\end{aligned}
$$

which introduces $L_{v}^{*}$ as follows:

$$
\frac{L_{v} \rho_{\text {ref }}}{p_{\text {ref }}}=31.25 \sim \varepsilon^{-1} L_{v}^{*} .
$$

These relations allow us to write $\Omega_{e}$ as follows:

$$
\begin{aligned}
\Omega_{e} & =\left(1+\frac{R_{v}}{R_{d}} q_{v}\right)^{R_{d} / c_{p_{*}}}\left(\frac{q_{v}}{q_{s}}\right)^{-R_{v} q_{t} / c_{p *}} \\
& =\left(1+R^{*} q_{v}\right)^{\Gamma * \varepsilon\left(1+c_{p}^{*} \varepsilon^{-1} q_{t}\right)^{-1}}\left(\frac{q_{v}}{q_{s}}\right)^{-R^{*} \Gamma^{*} \varepsilon q_{v}\left(1+c_{p}^{*} \varepsilon^{-1} q_{t}\right)^{-1}} .
\end{aligned}
$$

All of which may be combined with the expression for $\theta_{e}[(\mathrm{~A} 1)]$ to derive a dimensionless equation of state expressed in terms of distinguished limits for nondimensional thermodynamic parameters: 


$$
\begin{aligned}
\rho \theta_{e}= & p^{\left[1-\Gamma^{*} \varepsilon\left(1+\varepsilon^{-1} c_{p}^{*} q_{t}\right)^{-1}\right]}\left(1+q_{t}\right)\left(1+R^{*} q_{v}\right)^{\left[-1+\Gamma \varepsilon\left(1+\varepsilon^{-1} c_{p}^{*} q_{t}\right)^{-1}\right]} \\
& \times\left(\frac{q_{v}}{q_{s}}\right)^{-R^{*} \Gamma^{*} \varepsilon q_{v}\left(1+c_{p}^{*} \varepsilon^{-1} q_{t}\right)^{-1}} \exp \left[\frac{L_{v}^{*} \Gamma^{*}}{\left(1+c_{p}^{*} \varepsilon^{-1} q_{t}\right)} \frac{\left(1+R^{*} q_{v}\right)}{\left(1+q_{t}\right)} \frac{\rho q_{v}}{p}\right] .
\end{aligned}
$$

\section{b. The $\theta_{e}$ expansions}

The expression for $\theta_{e}$ can be expanded in powers of $\delta$ as follows:

$\theta_{e}^{(0)}=1$,

$\theta_{e}^{(1)}=0$,

$\theta_{e}^{(2)}=0$,

$\theta_{e}^{(3)}=T^{(3)}+L_{v}^{*} \Gamma^{*} q_{v}^{(3)}$,

$\theta_{e}^{(4)}=T^{(4)}+L_{v}^{*} \Gamma^{*} q_{v}^{(4)}+\mathcal{Q}^{(4)}$,

$\theta_{e}^{(5)}=T^{(5)}+L_{v}^{*} \Gamma^{*} q_{v}^{(5)}+\mathcal{Q}^{(5)}$,

$\theta_{e}^{(6)}=T^{(6)}+L_{v}^{*} \Gamma^{*} q_{v}^{(6)}+\Gamma^{*} R^{*} q_{v}^{(4)}-L_{v}^{*} \Gamma^{*} c_{p}^{*} q_{v}^{(4)^{2}}+\mathcal{Q}^{(6)}$.
These can be further specified given an expansion for $T$ in (A8) as

$$
\begin{aligned}
T^{(i)}= & p^{(i)}-\rho^{(i)}+q_{t}^{(i)}-R^{*} q_{v}^{(i)} \quad \text { for } \quad i=3,4,5, \\
T^{(6)}= & p^{(6)}-\rho^{(6)}+q_{t}^{(6)}-R^{*} q_{v}^{(6)}-R^{*} q_{v}^{(3)} T^{(3)} \\
& -\left(T^{(3)}+R^{*} q_{v}^{(3)}\right) \rho^{(3)}+q_{t}^{(3)} p^{(3)}
\end{aligned}
$$

and the $\mathcal{Q}^{(i)}$ depend on the background moisture as follows:

$$
\begin{aligned}
\mathcal{Q}^{(4)}= & -L_{v}^{*} \Gamma^{*} c_{p}^{*} q_{t}^{(4)} q_{v}^{(3)} \\
\mathcal{Q}^{(5)}= & -L_{v}^{*} \Gamma^{*} c_{p}^{*}\left(q_{t}^{(4)} q_{v}^{(3)}+q_{t}^{(3)} q_{v}^{(4)}-c_{p}^{*} q_{t}^{(3)} q_{v}^{(3)^{2}}\right) \\
& +\Gamma^{*} R^{*} q_{v}^{(3)}\left[1-\ln \left(\frac{q_{v}^{(3)}}{q_{s}^{(3)}}\right)\right]
\end{aligned}
$$

\section{c. Saturation vapor mixing ratio $q_{s}$}

The saturated water vapor mixing ratio $q_{s}$ is defined as

$$
q_{s}(T)=\frac{p_{s}^{\prime}(T)}{R^{*}\left[p^{\prime}-p^{\prime}(T)\right]}
$$

where $T$ is the temperature, $p$ is the air pressure, and $p_{\mathrm{s}}^{\prime}(T)$ is the equilibrium saturation vapor pressure given by Clausius-Clapeyron formula:

$$
\frac{d p_{s}^{\prime}}{d T}=\frac{L_{v}}{R_{v} T^{2}} p_{s}^{\prime}
$$

Both $R^{*}$ and $L_{v}$ are given as before. Integrating the Clausius-Clapeyron equation assuming that $L_{v}$ is independent of temperature yields the following approximation for $p_{s}^{\prime}$ :

$$
p_{s}^{\prime}=p_{s, \text { ref }}^{\prime} \exp \left[\frac{L_{v}}{R_{v} T_{\text {ref }}} \frac{\left(T^{\prime}-T_{\text {ref }}\right)}{T^{\prime}}\right]
$$

where $T_{0}=300 K$ and $p_{\mathrm{s}, \text { ref }}^{\prime}=3500 \mathrm{~kg} \mathrm{~m}^{-1} \mathrm{~s}^{-2}$. Defining $A=L_{v} / R_{v} \theta_{\text {ref }}=18.05 \approx \varepsilon^{-1} A^{*}$ and making use of the distinguished limits [(10)] yields

$$
p_{s}^{\prime}=p_{s, \text { ref }}^{\prime} \exp \left[\frac{A^{*}}{\varepsilon}\left(1-\frac{1}{T}\right)\right]
$$

where the dimensionless temperature is given by (A8). From this expression, and the additional distinguished limit $p_{s, \text { ref }}^{\prime} / p_{\text {ref }}=0.035 \approx \varepsilon^{3 / 2} p_{s}^{*}$, the following can be derived:

$$
q_{s}=\frac{\delta^{3} p_{s}^{*} \exp \left[\frac{A^{*}}{\delta^{2}}\left(1-\frac{1}{T}\right)\right]}{R^{*} p-\delta^{3} R^{*} p_{s}^{*} \exp \left[\frac{A^{*}}{\delta^{2}}\left(1-\frac{1}{T}\right)\right]},
$$


which concludes the derivation of (58) in the main text.

\section{APPENDIX B}

\section{Bulk Averaged Pressure Gradients}

Integrating the hydrostatic balance (38) and making use of the boundary conditions (37) implies that $p^{(0)}=1$, $p^{(1)}=0$, and $p^{(2)}=0$. It follows from the equation of state $[(8)]$ that

$$
\begin{aligned}
& \rho^{(0)}=\theta_{e}^{(0)}=p^{(0)}=1, \\
& \rho^{(1)}=\theta_{e}^{(1)}=p^{(1)}=0, \\
& \rho^{(2)}=\theta_{e}^{(2)}=p^{(2)}=0 .
\end{aligned}
$$

Using these expressions simplifies further expansions of the equation of state such that

$$
\begin{aligned}
\rho^{(3)}= & p^{(3)}+\varphi^{(3)}, \\
\rho^{(4)}= & p^{(4)}+\varphi^{(4)}, \\
\rho^{(5)}= & p^{(5)}+\varphi^{(5)}-\Gamma^{*} p^{(3)}, \\
\rho^{(6)}= & p^{(6)}+\varphi^{(6)}-\Gamma^{*} p^{(4)}-\rho^{(3)} \theta_{e}^{(3)}-R^{*}\left(\theta_{e}^{(3)}\right. \\
& \left.-\Gamma^{*} L_{v}^{*} q_{v}^{(3)}\right) q_{v}^{(3)}+\left(1+\Gamma^{*} c_{p}^{*}\right) q_{t}^{(3)} p^{(3)} \\
& +\left(\Gamma^{*} L_{v}^{*}-R^{*}\right) q_{v}^{(3)} \rho^{(3)},
\end{aligned}
$$

where

$$
\varphi^{(i)}=-\theta_{e}^{(i)}+q_{t}^{(i)}+\left(\Gamma^{*} L_{v}^{*}-R^{*}\right) q_{v}^{(i)}+\mathcal{Q}^{(i)},
$$

and $\mathcal{Q}^{(3)}=0$ and $\mathcal{Q}^{(i)}$ (for $\left.i=4,5,6\right)$ are given in appendix A by (A21)-(A23).

The above expressions for $\rho^{(i)}$ through $i=6$ allow us, through integration of (39), to derive expressions for $p$ through $i=9$, these being

$$
\begin{aligned}
p^{(3)}= & -\eta, \\
p^{(4)}= & 0, \\
p^{(5)}= & 0, \\
p^{(6)}= & \frac{\eta^{2}}{2}+\varphi^{(3)}(H-\eta), \\
p^{(7)}= & \varphi^{(4)}(H-\eta), \\
p^{(8)}= & \varphi^{(5)}(H-\eta)+p_{g}-\frac{1}{2} \Gamma^{*} \eta^{2}, \\
p^{(9)}= & \left(q_{t}^{(6)}-\theta_{e}^{(6)}\right)(H-\eta)+\left(\Gamma^{*} L_{v}^{*}-R^{*}\right) \\
& \times \int_{\eta}^{H} q_{v}^{(6)} d \eta-\frac{\eta^{3}}{6}+\frac{1}{2} \varphi^{(3)}(H-\eta)^{2} \\
& +\mathcal{T}_{1}(H-\eta)+\mathcal{T}_{2}\left(H^{2}-\eta^{2}\right),
\end{aligned}
$$

where

$$
\begin{aligned}
\mathcal{T}_{1}= & \mathcal{Q}^{(6)}-\left(\theta_{e}^{(3)}-L_{v}^{*} \Gamma^{*} q_{v}^{(3)}\right)\left(\varphi^{(3)}+R^{*} q_{v}^{(3)}\right) \\
& -R^{*} \varphi^{(3)} q_{v}^{(3)}, \mathcal{T}_{2}=\theta_{e}^{(3)}-\left(L_{v}^{*} \Gamma^{*}-R^{*}\right) q_{v}^{(3)} \\
& +\left(1+\Gamma^{*} c_{p}^{*}\right) q_{t}^{(3)} .
\end{aligned}
$$

Based on the above, the pressure gradients are written as a series expansion in $\delta$ such that

$$
\begin{aligned}
\nabla_{X} p= & \delta^{6}\left(\varphi^{(3)} \nabla_{X} H\right)+\delta^{7}\left(\varphi^{(4)} \nabla_{X} H\right)+\delta^{(9)}\left(\varphi^{(5)} \nabla_{X} H+\nabla_{X} p_{g}\right)+\delta^{9}\left\{\left(q_{t}^{(6)}-\theta_{e}^{(6)}+\mathcal{T}_{1}\right) \nabla_{X} H+(H-\eta)\left[\varphi^{(3)} \nabla_{X} H\right.\right. \\
& \left.\left.+\nabla_{X}\left(q_{t}^{(6)}-\theta_{e}^{(6)}\right)\right]+\mathcal{T}_{2} H \nabla_{X} H+\left(\Gamma^{*} L_{v}^{*}-R^{*}\right) \nabla_{X} \int_{\eta}^{H} q_{v}^{(6)} d \eta\right\}+O\left(\delta^{10}\right) .
\end{aligned}
$$

Integrating (B16) over the vertical provides the desired expression for the vertically integrated pressure gradient. This is straightforward once terms involving the vertical integral of the water vapor terms of sixth order and greater are evaluated. These contribute to the expression for $p^{(9)}$. As per our definition, the water vapor mixing ratio is given by

$$
q_{v}= \begin{cases}q_{s}, & \eta \geq \eta_{c} \\ q_{t}, & \eta<\eta_{c}\end{cases}
$$

where $\eta_{c}$ is the condensation height (an expression for it is derived in section 5a). In section $4 \mathrm{~b}$ we argued that $q_{s}$ varies with $\eta$ already at order six and in section 5a showed that asymptotic expansion of saturation mixing ratio yields $q_{s}^{(6)}=\beta_{0}+\beta_{1} \eta$. Hence, for

$$
\begin{aligned}
\int_{\eta}^{H} q_{v} d \eta= & \delta^{4} q_{t}^{(4)}(H-\eta)+\delta^{5} q_{t}^{(5)}(H-\eta) \\
& +\delta^{6} \int_{\eta}^{H} q_{v}^{(6)} d \eta+\cdots
\end{aligned}
$$

we can evaluate the last term as follows: 


$$
\int_{\eta}^{H} q_{v}^{(6)} d \eta= \begin{cases}\beta_{0}(H-\eta)+\left(\frac{H^{2}}{2}-\frac{\eta^{2}}{2}\right), & \eta \geq \eta_{c} \\ \beta_{0}\left(H-\eta_{c}\right)+\beta_{1}\left(\frac{H^{2}}{2}-\frac{\eta_{c}^{2}}{2}\right)+q_{t}^{(6)}\left(\eta_{c}-\eta\right), & \eta<\eta_{c} .\end{cases}
$$

Taking the gradient and integrating over the boundary layer provides the desired expression:

$$
\begin{aligned}
\int_{0}^{H}\left(\nabla_{X} \int_{\eta^{\prime}}^{H} q_{v}^{(6)} d \eta^{\prime}\right) d \eta= & \beta_{0} H \nabla_{X} H+\beta_{1} H^{2} \nabla_{X} H \\
& +\frac{\beta_{1}}{6} \nabla_{X} \eta_{c}^{3} .
\end{aligned}
$$

Given the expansion for $H$ assumed in section $4 \mathrm{~b}$, this completes the derivation of the vertically averaged pressure gradients.

\section{APPENDIX C}

\section{Dispersion Relation}

We linearize (101)-(104) about the steady-state solution described in section $6 \mathrm{~b}$ and neglect the Coriolis effects, $y$ variations in the flow variables, and the $y$ component of velocity. The linearized equations are

$$
\begin{array}{r}
\frac{\partial h^{\prime}}{\partial t}+u^{(0)} \frac{\partial h^{\prime}}{\partial x}+H^{(0)} \frac{\partial u^{\prime}}{\partial x}+\mathcal{C}_{1} u^{\prime}=0, \\
\frac{\partial u^{\prime}}{\partial t}+u^{(0)} \frac{\partial u^{\prime}}{\partial x}+\varphi^{(5)} \frac{\partial h^{\prime}}{\partial x}+\mathcal{C}_{2} \frac{\partial \theta_{e}^{\prime}}{\partial x}+\mathcal{C}_{3} \frac{\partial \eta_{c}^{\prime}}{\partial x}=0, \\
\frac{\partial \theta_{e}^{\prime}}{\partial t}+u^{(0)} \frac{\partial \theta_{e}^{\prime}}{\partial x}+\mathcal{C}_{4} \eta_{c}^{\prime}+\mathcal{C}_{5} u^{\prime}=0, \\
\frac{\partial \eta_{c}^{\prime}}{\partial t}+u^{(0)} \frac{\partial \eta_{c}^{\prime}}{\partial x}+\mathcal{C}_{6} \eta_{c}^{\prime}+\mathcal{C}_{7} u^{\prime}=0,
\end{array}
$$

where the prime denotes perturbations from the steadystate solutions and

$$
\begin{aligned}
& \mathcal{C}_{1}=-4 \mathcal{E}_{h} \bar{u}^{3} ; \quad \mathcal{C}_{2}=-\frac{H^{(0)}}{2} ; \quad \mathcal{C}_{3}=\frac{\beta_{1}}{2 H^{(0)}}\left[H^{(0)^{2}}+\left(\Gamma^{*} L_{v}^{*}-R^{*}\right) \bar{\eta}^{2}\right] ; \quad \mathcal{C}_{4}=\frac{\beta_{1} a^{*}}{H^{(0)}} \Delta F\left(H^{(0)}-\bar{\eta}_{c}\right) ; \quad \mathcal{C}_{5}=-4 \mathcal{E}_{\theta} \bar{u}^{3} ; \\
& \mathcal{C}_{6}=\frac{\alpha_{p}}{H^{(0)}} \mathcal{P}^{*}\left(H^{(0)}-\bar{\eta}_{c}\right)^{\left(2 \alpha_{p}-1\right)} ; \quad \mathcal{C}_{7}=-4 \mathcal{E}_{\eta} \bar{u}^{3} ; \quad \mathcal{E}_{h}=\frac{C_{E}^{*}}{H^{(0)}} \varphi^{(5)^{-3 / 2}} ; \quad \mathcal{E}_{\theta}=\frac{\mathcal{E}_{h}}{H^{(0)}} \Delta \Theta_{e} ; \quad \mathcal{E}_{\eta}=\frac{\mathcal{E}_{h}}{\beta_{1} H^{(0)}} \Delta Q_{t} ;
\end{aligned}
$$

$\bar{u}$ and $\bar{\eta}_{c}$ denote the steady-state velocity $u^{(1)}$ and cloudbase height $\eta_{c}$, respectively; $\Delta \Theta_{e}$ is the cloud-top jump in equivalent potential temperature; and $\Delta Q_{t}$ is the cloud top jump in total water mixing ratio.

The linear stability analysis performed here is based on the classical plane wave solutions to the equations having the form

$$
\left(\begin{array}{c}
h^{\prime} \\
u^{\prime} \\
\theta_{e}^{\prime} \\
\eta_{c}^{\prime}
\end{array}\right)=\left(\begin{array}{c}
\widehat{h} \\
\widehat{u} \\
\widehat{\theta}_{e} \\
\widehat{\eta}_{c}
\end{array}\right) \exp [i(k x-\omega t)]
$$

where $\omega$ denotes frequency and $k$ is the wavenumber in the $x$ direction. With these substitutions, (C1)-(C4) become

$$
\mathbf{M} \hat{\mathbf{u}}=0
$$

in which

$$
\mathbf{M}=\left(\begin{array}{cccc}
-i \sigma & i H^{(0)} k+\mathcal{C}_{1} & 0 & 0 \\
i \varphi^{(5)} k & -i \sigma & i \mathcal{C}_{2} k & i \mathcal{C}_{3} k \\
0 & \mathcal{C}_{5} & -i \sigma & \mathcal{C}_{4} \\
0 & \mathcal{C}_{7} & 0 & \mathcal{C}_{6}-i \sigma
\end{array}\right) \text { and } \quad \hat{\mathbf{u}}=\left(\begin{array}{c}
\hat{h} \\
\hat{u} \\
\hat{\theta}_{e} \\
\hat{\eta}_{c}
\end{array}\right)
$$

$\sigma=\omega-u^{(0)} k$ is the intrinsic frequency. The necessary condition for a nontrivial solution of the resulting system requires that $\operatorname{det}(\mathbf{M})=0$. This condition, called the dispersion relation, expresses the link between frequency $\omega$ and wavenumber $k$. The dispersion relation is is given:

$$
\begin{aligned}
\sigma^{4} & +i \mathcal{C}_{6} \sigma^{3}+\left[i\left(\mathcal{C}_{7} \mathcal{C}_{3}+\mathcal{C}_{5} \mathcal{C}_{2}+\mathcal{C}_{1} \varphi^{(5)}\right) k-\varphi^{(5)} H^{(0)} k^{2}\right] \sigma^{2} \\
& +\left[\left(\mathcal{C}_{7} \mathcal{C}_{4} \mathcal{C}_{2}-\mathcal{C}_{6} \mathcal{C}_{5} \mathcal{C}_{2}-\mathcal{C}_{6} \mathcal{C}_{1} \varphi^{(5)}\right) k\right. \\
& \left.-\varphi^{(5)} H^{(0)} \mathcal{C}_{6} k^{2}\right] \sigma=0
\end{aligned}
$$

\section{REFERENCES}

Biello, J. A., and A. J. Majda, 2005: A new multiscale model for the Madden-Julian oscillation. J. Atmos. Sci., 62, 1694-1721. 
Comstock, K., C. S. Bretherton, and S. E. Yuter, 2005: Mesoscale variability and drizzle in southeast Pacific stratocumulus. J. Atmos. Sci., 62, 3792-3807.

Dolaptchiev, S., and R. Klein, 2009: Planetary geostrophic equations for the atmosphere with evolution of the barotropic flow. Dyn. Atmos. Oceans, 46, 46-61.

Emanuel, K. A., 1994: Atmospheric Convection. Oxford University Press, 580 pp.

Faloona, I., and Coauthors, 2005: Observations of entrainment in eastern Pacific marine stratocumulus using three conserved scalars. J. Atmos. Sci., 62, 3268-3285.

Klein, R., 2004: An applied mathematical view of meteorological modeling. SIAM Proc. Appl. Math., 116, 177-219.

, 2010: Scale-dependent models for atmospheric flows. Annu. Rev. Fluid Mech., 42, 249-274.

— , and A. J. Majda, 2006: Systematic multiscale models for deep convection on mesoscales. Theor. Comput. Fluid Dyn., 20, 525-551.

— E. Mikusky, and A. Z. Owinoh, 2005: Multiple scale asymptotics for atmospheric flow. Proc. Fourth European Congress of Mathematics, Stockholm, Sweden, European Mathematical Society, 201-220.

Lenderink, G., and A. P. Siebesma, 2004: On the role of drizzle in stratocumulus and its implications for large-eddy simulation. Quart. J. Roy. Meteor. Soc., 130, 3429-3434.

Lilly, D. K., 1968: Models of cloud-topped mixed layers under a strong inversion. Quart. J. Roy. Meteor. Soc., 94, 292-309.

Lindzen, R. S., and S. Nigam, 1987: On the role of sea surface temperature gradients in forcing low-level winds and convergence in the tropics. J. Atmos. Sci., 44, 2418-2436.

Majda, A. J., and R. Klein, 2003: Systematic multiscale models for the tropics. J. Atmos. Sci., 60, 393-408.

Mikusky, E., 2007: On the structure of concentrated atmospheric vortices in the gradient wind regime and their motion on synoptic scales. Doctoral thesis, Universität Hamburg, 195 pp.
Moeng, C.-H., 2000: Entrainment rate, cloud fraction, and liquid water path of PBL stratocumulus clouds. J. Atmos. Sci., 57, 3627-3643.

_ , P. P. Sullivan, and B. Stevens, 1999: Including radiative effects in an entrainment rate formula for buoyancy-driven PBLs. J. Atmos. Sci., 56, 1031-1049.

Pawlowska, H., and J.-L. Brenguier, 2003: An observational study of drizzle formation in stratocumulus clouds for general circulation model (GCM) parameterization. J. Geophys. Res., 108, 8630, doi:10.1029/2002JD002679.

Pelly, J. L., and S. E. Belcher, 2001: A mixed-layer model of the well-mixed stratocumulus-topped boundary layer. Bound.Layer Meteor., 100, 171-187.

Savic-Jovcic, V., and B. Stevens, 2007: The structure and mesoscale organization of precipitating stratocumulus. J. Atmos. Sci., 65, $1587-1605$

Schubert, W. H., J. S. Wakefield, E. J. Steiner, and S. K. Cox, 1979: Marine stratocumulus convection. Part II: Horizontally inhomogeneous solutions. J. Atmos. Sci., 36, 1308-1324.

Stevens, B., 2002: Entrainment in stratocumulus-topped mixed layers. Quart. J. Roy. Meteor. Soc., 128, 2663-2690.

—, W. R. Cotton, G. Feingold, and C.-H. Moeng, 1998: Largeeddy simulations of strongly precipitating, shallow, stratocumulustopped boundary layers. J. Atmos. Sci., 55, 3616-3638.

— , and Coauthors, 2003: On entrainment rates in nocturnal marine stratocumulus. Quart. J. Roy. Meteor. Soc., 129, 34693492.

_ - and Coauthors, 2005: Evaluation of large-eddy simulations via observations of nocturnal marine stratocumulus. Mon. Wea. Rev., 133, 1443-1462.

Strang, E. J., and H. J. S. Fernando, 2001: Entrainment and mixing in stratified shear flows. J. Fluid Mech., 428, 349-386.

van Zanten, M. C., B. Stevens, G. Vali, and D. Lenschow, 2005 : Observations of drizzle in nocturnal marine stratocumulus. J. Atmos. Sci., 62, 88-106. 